\title{
Moving crack with a contact zone at interface of magnetoelectroelastic bimaterial
}

\author{
P. $\mathrm{Ma}^{1}$, R.K.L Su${ }^{1, *}$, W.J. Feng, ${ }^{2, *}$ \\ ${ }^{1}$ Department of Civil Engineering, The University of Hong Kong, Hong Kong, PR China \\ ${ }^{2}$ Department of Engineering Mechanics, Shijiazhuang Tiedao University, Shijiazhuang 050043, PR China
}

\begin{abstract}
The plane-strain problem of a moving crack at the interface of two dissimilar magnetoelectroelastic (MEE) materials is investigated. Assuming that the crack moves at a constant speed in the subsonic regime, a fracture analysis of a finite crack under concentrated loading imposed onto the crack face is first carried out. By applying magnetoelectric (ME) permeable boundary conditions at the crack face, a combined Dirichlet-Riemann problem is formulated and solved analytically. The expressions for the fracture parameters, including the relative length of the contact zone and field intensity factors (FIFs), are obtained in the analytical form. A crack of a semi-infinite length with a contact zone under concentrated loading is further presented as a specific case examined with the obtained solution. Then a moving crack of finite length at the interface under remote mix-mode loading is also analyzed and the corresponding fracture parameters are presented in an analytical form. Finally, numerical examples are provided for the material combination of barium titanate-cobalt ferrite composites to examine the influence of the speed of the moving crack, poling direction, material volume fraction, load position and
\end{abstract}

\footnotetext{
* Corresponding author. Tel: +852 2859 2648, fax: +852 25595337 (R.K.L. Su);

E-mail address: mapengsjz@163.com (P. Ma); klsu@hku.hk (R.K.L. Su); wjfeng9999@126.com (W.J. Feng).
} 
load ratio on the fracture parameters, from which some new and interesting conclusions related to the crack model in this study are drawn.

Keywords: Moving crack; Interface crack; Stress intensity factor; Contact zone; Magnetoelectroelastic materials

\section{Introduction}

Magnetoelectroelastic (MEE) laminated composites made of piezoelectric and piezomagnetic materials have become promising candidates as the core components of multifunctional magnetoelectric (ME) energy conversion devices, for example, sensors, actuators, transducers, and surface acoustic wave (SAW) devices, owing to their unique MEE coupling effect. However, during the service of these MEE components, cracks or flaws usually develop at the interface of dissimilar materials, which will greatly reduce their performance and remaining life, especially in dynamic loading environments. Therefore, it is important to examine interface cracks and their propagation in MEE materials. More recently, interface crack problems in MEE bimaterial have received much attention [1-5].

In practical engineering, dynamic interface cracks and their propagation are more problematic due to external loading conditions. One of the most important topics in dynamic fracture mechanics is examining transient response. The transient response of anti-plane cracks at the interface of MEE material was first investigated by researchers who used Laplace- and Fouriertransforms [6-10]. As for the problem of plane cracks at the interface, Zhong et al. [11] studied the transient response of two limited permeable collinear cracks in a homogeneous MEE body under impact loadings. Chen [12] established a dynamic contour integral for cracked magnetoelectro-thermo-elastic (METE) materials with the use of Helmholtz free-energy and the 
fundamental principles of thermodynamics, and pointed out that the dynamic contour integral could be employed as a physically sound criterion for the dynamic fracture analysis of METE solids. Hu and Chen [13] considered the problem of cracks due to the pre-curving of an MEE strip subjected to impact loadings. They applied magnetoelectric (ME) impermeable and permeable boundary conditions at the crack face and obtained the hoop stress intensity factors. It was shown that according to the criterion of maximum hoop stress, negative and positive electric and/or magnetic loadings can inhibit or promote the development of an ME impermeable crack, respectively. Feng et al. [14] investigated the transient response of an interface crack between two MEE layers under Mode-I impact loading. They demonstrated that a dynamic energy release rate (ERR) is not oscillating, and the impacts of magnetic or electric loading have the same effects on the dynamic ERR as those in a static case.

Another important issue in dynamic fracture mechanics is the problem of moving cracks, which can be traced back to the work by Yoffe [15] in relation to elastic material and then continued by Willis [16], Atkinson [17] and other pioneering researchers. The moving crack model has also been extended to analyze the fracture behaviors of homogeneous MEE materials [18-23] and MEE bimaterials [24-26]. Among the various studies, Zhong and Li [24] studied a Yoffe moving crack at the interface of MEE bimaterial based on the limited permeable ME crack-face boundary conditions. They concluded that the speed of the moving crack has no influence on the stress ahead of the tip of the moving crack. Instead, it has an apparent effect on the distribution of the stress around the tip of the moving crack. By assuming zero electric and magnetic potentials in the area of the crack, Chen et al. [25] considered the propagation of a semi-infinite crack at the interface between piezoelectric and piezomagnetic solids. They observed that in determining the dynamic fracture parameters, the B-G waves play an important role in the considered material combinations. Using the Fourier-transform method, Hu et al. [26] investigated an ME permeable 
Dugdale-type of moving crack at the interface of an MEE bimaterial. They stated that the extended stresses are no longer singular and crack sliding displacement is dependent on the applied load, material properties and velocity of the moving crack. However, the aforementioned fracture analysis of the interface is only limited to anti-plane problems. In-plane fracture analysis of cracks at the interface has not been reported yet owing to the complexities of the mathematics and physics involved.

On the other hand, interface crack models with a contact zone have been proposed and applied to addressing problems of interface fracture [27-29]. It has been shown that a real contact zone exists and influences the fracture behavior at the crack tip. Therefore, in this study, we investigate the fracture characteristics of an MEE bimaterial by applying an interface crack model of a moving crack with a contact zone. The speed of the moving crack is assumed to be slower than the minimum Rayleigh wave speed of the MEE bimaterial. Three cases are considered, namely, cracks of finite and semi-infinite lengths under a concentrated load, and a crack of a finite length under a uniform mix-mode load. The expressions for determining the contact zone and FIFs are obtained. Numerical results are provided for the material combination of barium titanate-cobalt ferrite $\left(\mathrm{BaTiO}_{3}-\mathrm{CoFe}_{2} \mathrm{O}_{4}\right)$ composites to investigate the effects of a number of factors, including speed of the crack, poling direction, material volume fraction, and load ratio and position, on the fracture parameters, which has not been carried out in previous research work.

\section{Basic relations of MEE bimaterial by using moving coordinate system}

According to Huang et al. [30], the dynamic constitutive equations for an MEE solid in a fixed coordinate system $\left(X_{1}, X_{2}, X_{3}\right)$ can be written in the form: 


$$
\begin{aligned}
& \left\{\begin{array}{l}
\sigma_{i j}=c_{i j k l} u_{k, l}-e_{l i j} \varphi_{, l}-h_{l i j} \phi_{, l} \\
D_{i}=e_{i k l} u_{k, l}+\alpha_{i l} \varphi_{, l}+d_{i l} \phi_{, l} \\
B_{i}=h_{i k l} u_{k, l}+d_{i l} \varphi_{, l}+\mu_{i l} \phi_{, l}
\end{array}\right. \\
& \left\{\begin{array}{l}
\left(c_{i j k l} u_{k}+e_{l i j} \varphi+h_{l i j} \phi\right)_{, l i}=\rho \frac{\partial^{2} u_{j}}{\partial t^{2}} \\
\left(e_{i k l} u_{k}-\alpha_{i l} \varphi-d_{i l} \phi\right)_{, l i}=0 \\
\left(h_{i k l} u_{k}-d_{i l} \varphi-\mu_{i l} \phi\right)_{, l i}=0
\end{array}\right.
\end{aligned}
$$

where $\sigma_{i j}, D_{i}, B_{i}$ are the components of the stresses, electric displacements and magnetic inductions, respectively; $u_{i}, \varphi, \phi$ are the mechanical displacement components, and electric and magnetic potentials, respectively. $c_{i j k l}, e_{i k l}, h_{i k l}, d_{i l}$ are the elastic, piezoelectric, piezomagnetic, and electromagnetic constants, respectively; $\alpha_{i l}, \mu_{l i}$ are the dielectric permittivity and magnetic permeability, respectively, and $\rho$ is the material density. $i, j, k, s$ range in $\{1,2,3\}$, the repeated indexes imply summation, and the comma stands for the differentiation with respect to the corresponding coordinate variables.

It is assumed that the speed of the crack $v$ is less than the minimum Rayleigh wave speed for a bimaterial system, and herein called the critical surface wave speed $c_{\mathrm{cr}}$. Therefore, the subsonic regime is investigated [31, 32]. In this case, the Lekhnitskii-Eshelby-Stroh formalism of generalized complex potentials can also be applied to the problem in this study. Here, we consider an in-plane fracture problem, in which all the fields are independent of the coordinate $x_{2}$, and the displacement $u_{2}$ decouples in the $\left(x_{1}, x_{3}\right)$-plane from the components $\mathbf{U}=\left\{u_{1}, u_{3}, \varphi, \phi\right\}^{\mathrm{T}}$. 
Assuming that the crack moves along the material interface, the following coordinate transformation $x_{1}=X_{1}-v t, x_{2}=X_{2}, x_{3}=X_{3}$ is performed, where $v$ is the speed of the crack tip. In the moving coordinate system, Eq. (2) can be rewritten as:

$\mathbf{Q} \mathbf{U}_{, 11}+\left(\mathbf{R}+\mathbf{R}^{\mathrm{T}}\right) \mathbf{U}_{, 13}+\mathbf{T} \mathbf{U}_{33}=0$

where

$\mathbf{Q}=\left(\begin{array}{ccc}\mathbf{Q}_{0} & \mathbf{e}_{11} & \mathbf{h}_{11} \\ \mathbf{e}_{11}^{\mathrm{T}} & -\alpha_{11} & -d_{11} \\ \mathbf{h}_{11}^{\mathrm{T}} & -d_{11} & -\mu_{11}\end{array}\right), \mathbf{R}=\left(\begin{array}{ccc}\mathbf{R}_{0} & \mathbf{e}_{31} & \mathbf{h}_{31} \\ \mathbf{e}_{13}^{\mathrm{T}} & -\alpha_{13} & -d_{13} \\ \mathbf{h}_{13}^{\mathrm{T}} & -d_{13} & -\mu_{13}\end{array}\right), \mathbf{T}=\left(\begin{array}{ccc}\mathbf{T}_{0} & \mathbf{e}_{33} & \mathbf{h}_{33} \\ \mathbf{e}_{33}^{\mathrm{T}} & -\alpha_{33} & -d_{33} \\ \mathbf{h}_{33}^{\mathrm{T}} & -d_{33} & -\mu_{33}\end{array}\right)$

$\left(\mathbf{Q}_{0}\right)_{j k}=c_{1 j k 1}-\rho v^{2} \delta_{j k},\left(\mathbf{R}_{0}\right)_{j k}=c_{1 j k 3},\left(\mathbf{T}_{0}\right)_{j k}=c_{3 j k 3},\left(\mathbf{e}_{i j}\right)_{m}=e_{i j m},\left(\mathbf{h}_{i j}\right)_{m}=h_{i j m}$

and $\delta_{j k}$ is the Kronecker delta.

By using the Lekhnitskii-Eshelby-Stroh formalism and applying this formalism to MEE materials, a general solution with Eq. (3) can be presented in the forms [1]

$$
\begin{aligned}
& \mathbf{U}=\mathbf{A f}(z)+\overline{\mathbf{A}} \overline{\mathbf{f}}(\bar{z}) \\
& \mathbf{t}=\mathbf{B f}^{\prime}(z)+\overline{\mathbf{B}} \overline{\mathbf{f}}^{\prime}(\bar{z})
\end{aligned}
$$

where $\mathbf{U}=\left\{u_{1}, u_{3}, \varphi, \phi\right\}^{\mathrm{T}}, \mathbf{t}=\left\{\sigma_{31}, \sigma_{33}, D_{3}, B_{3}\right\}^{\mathrm{T}}$ (the superscript 'T' stands for the transposed matrix), $\mathbf{A}=\left\{\mathbf{A}_{1}, \mathbf{A}_{2}, \mathbf{A}_{3}, \mathbf{A}_{4}\right\}^{\mathrm{T}} ; \mathbf{f}(z)=\left\{\mathbf{f}_{1}\left(z_{1}\right), \mathbf{f}_{2}\left(z_{2}\right), \mathbf{f}_{3}\left(z_{3}\right), \mathbf{f}_{4}\left(z_{4}\right)\right\}^{\mathrm{T}}$ in Eqs. (5) and (6) is an arbitrary analytic vector function with four components; $z_{j}=x_{1}+p_{j} x_{3}(j=1,2,3,4)$. For a fixed $j, p_{j}$ and $\mathbf{A}_{j}=\left\{a_{1 j}, a_{2 j}, a_{3 j}, a_{4 j}\right\}^{\mathrm{T}}$ are respectively an eigenvalue and an eigenvector of the system

$$
\left\{\mathbf{Q}+p_{j}\left(\mathbf{R}+\mathbf{R}^{\mathrm{T}}\right)+p_{j}^{2} \mathbf{T}\right\} \mathbf{A}_{j}=0
$$


The $4 \times 4$ matrix $\mathbf{B}$ can be determined by using the formula $\mathbf{B}=\mathbf{R}^{\mathrm{T}} \mathbf{A}+\mathbf{T A P}$ with $\mathbf{P}=\operatorname{diag}\left\{p_{1}, p_{2}, p_{3}, p_{4}\right\}$. The prime (') denotes differentiation with respect to the argument, and the overbar stands for the complex conjugate.

A comparison between Eqs. (3)-(7) with the related equations in Section 2 of the paper by Herrmann and Loboda [33] which deal with a stationary crack shows that they are formally similar, which has been mentioned in many papers on subsonic crack propagation. Therefore, the method developed in Herrmann and Loboda [33] for a static interface crack can also be applied to carry out a study on a moving crack on MEE bimaterial.

Using this approach for a moving crack at the interface of a piezoelectric bimaterial by Herrmann et al. [32], one arrives at the following expressions:

$$
\begin{aligned}
& {\left[\mathbf{U}^{\prime}\left(x_{1}\right)\right]=\mathbf{W}^{+}\left(x_{1}\right)-\mathbf{W}^{-}\left(x_{1}\right)} \\
& \mathbf{t}^{(1)}\left(x_{1}, 0\right)=\mathbf{G} \mathbf{W}^{+}\left(x_{1}\right)-\overline{\mathbf{G}} \mathbf{W}^{-}\left(x_{1}\right)
\end{aligned}
$$

where $\mathbf{W}(z)=\left\{W_{1}(z), W_{3}(z), W_{4}(z), W_{5}(z)\right\}^{\mathrm{T}}$ is an introduced unknown vector function, which is analytic in the whole plane with a cut along the crack region $(c, b)$ and $\mathbf{W}^{ \pm}\left(x_{1}\right)=\mathbf{W}\left(x_{1} \pm \mathrm{i} 0\right) ; \mathbf{G}=\mathbf{B}^{(1)} \mathbf{D}^{-1}, \quad$ where $\mathbf{D}=\mathbf{A}^{(1)}-\overline{\mathbf{L}} \mathbf{B}^{(1)}, \mathbf{L}=\mathbf{A}^{(2)}\left(\mathbf{B}^{(2)}\right)^{-1}$ and superscripts (1) and (2) denote the upper and lower materials, respectively; the prime denotes differentiation with respect to the argument. For the transversely isotropic MEE bimaterials in this study, which can be arbitrarily oriented with respect to the crack surface, the matrix $\mathbf{G}$ has the following structure:

$$
\mathbf{G}=\left(\begin{array}{cccc}
\mathrm{i} g_{11} & g_{13} & g_{14} & g_{15} \\
g_{31} & \mathrm{i} g_{33} & g_{34} & g_{35} \\
g_{41} & g_{43} & \mathrm{i} g_{44} & g_{45} \\
g_{51} & g_{53} & g_{54} & \mathrm{i} g_{55}
\end{array}\right)
$$


where all $g_{i i}(i=1,3,4,5)$ are real, and $g_{i j}(i, j=1,3,4,5 ; i \neq j)$ are complex; $g_{i j}=-\bar{g}_{j i}$ holds true and $\mathrm{i}=\sqrt{-1}$. It is worth noting that if the bimaterial system is poled in the $x_{3}$-direction, the structure of matrix $\mathbf{G}$ in Eq. (10) will be reduced to the one presented in the investigations by Herrmann et al. [27] and Feng et al. [29].

Using the ME permeable conditions at the crack region and the absence of load at infinity, $W_{4}=W_{5}=0$ holds true in the whole plane. Thus, the extended stresses at the interface can be presented as:

$$
\left\{\begin{array}{l}
\sigma_{13}^{(1)}\left(x_{1}, 0\right)=\mathrm{i} g_{11} W_{1}^{+}\left(x_{1}\right)+\mathrm{i} g_{11} W_{1}^{-}\left(x_{1}\right)+g_{13} W_{3}^{+}\left(x_{1}\right)-\bar{g}_{13} W_{3}^{-}\left(x_{1}\right) \\
\sigma_{33}^{(1)}\left(x_{1}, 0\right)=g_{31} W_{1}^{+}\left(x_{1}\right)-\bar{g}_{31} W_{1}^{-}\left(x_{1}\right)+\mathrm{i} g_{33} W_{3}^{+}\left(x_{1}\right)+\mathrm{i} g_{33} W_{3}^{-}\left(x_{1}\right) \\
D_{3}^{(1)}\left(x_{1}, 0\right)=g_{41} W_{1}^{+}\left(x_{1}\right)-\bar{g}_{41} W_{1}^{-}\left(x_{1}\right)+g_{43} W_{3}^{+}\left(x_{1}\right)-\bar{g}_{43} W_{3}^{-}\left(x_{1}\right) \\
B_{3}^{(1)}\left(x_{1}, 0\right)=g_{51} W_{1}^{+}\left(x_{1}\right)-\bar{g}_{51} W_{1}^{-}\left(x_{1}\right)+g_{53} W_{3}^{+}\left(x_{1}\right)-\bar{g}_{53} W_{3}^{-}\left(x_{1}\right)
\end{array}\right.
$$

Combining the first and second formulas of Eq. (11), one arrives at the following expressions at the interface:

$\sigma_{33}^{(1)}\left(x_{1}, 0\right)+\mathrm{i} m_{j} \sigma_{13}^{(1)}\left(x_{1}, 0\right)=t_{j}\left\{\Omega_{j}^{+}\left(x_{1}\right)+\gamma_{j} \Omega_{j}^{-}\left(x_{1}\right)\right\}, j=1,3$

where

$$
\begin{aligned}
& \Omega_{j}(z)=W_{1}(z)+i_{j} W_{3}(z), m_{1,3}=\frac{\left(g_{13}+g_{31}\right) \pm \sqrt{\left(g_{13}+g_{31}\right)^{2}+4 g_{33} g_{11}}}{2 g_{11}} \\
& t_{j}=g_{31}-g_{11} m_{j}, s_{j}=\frac{g_{33}+g_{13} m_{j}}{t_{j}}, \gamma_{j}=\frac{g_{13}-g_{11} m_{j}}{t_{j}}
\end{aligned}
$$

and the constants $m_{1,3}$ and $s_{1,3}$ are complex while $t_{1,3}$ and $\gamma_{1,3}$ are real. They have the following relations, $s_{1,3}=-m_{1,3}$ and $\gamma_{1} \gamma_{3}=1$.

Eq. (8) leads to the following expressions related to the derivatives of the displacement jumps 
$\left[u_{1}^{\prime}\left(x_{1}\right)\right]+$ is $_{j}\left[u_{3}^{\prime}\left(x_{1}\right)\right]=\Omega_{j}^{+}\left(x_{1}\right)-\Omega_{j}^{-}\left(x_{1}\right), j=1,3$

where the prime denotes differentiation with respect to the argument. In the following sections, the focus will be on the case of $j=1$ only since it is sufficient enough to determine the unknowns.

\section{Moving crack of finite length at interface under concentrated load}

A bimaterial composed of two dissimilar MEE semi-infinite planes $x_{3}>0$ and $x_{3}<0$ with material properties defined by the following material constants $c_{i j k s}^{(1)}, e_{i k s}^{(1)}, h_{i k s}^{(1)}, d_{i s}^{(1)}, \alpha_{i s}^{(1)}, \mu_{s i}^{(1)}$ and $c_{i j k s}^{(2)}, e_{i k s}^{(2)}, h_{i k s}^{(2)}, d_{i s}^{(2)}, \alpha_{i s}^{(2)}, \mu_{s i}^{(2)}$, respectively, is considered (Fig. 1), where superscripts (1) and (2) denote the upper and lower materials, respectively. It is assumed that the upper material is poled in the $x_{3}$-direction and the lower material can be arbitrarily oriented with respect to the surface of the crack (angle $\beta_{0}$ ). An ME permeable crack situated in $x_{1} \in(c, b), x_{3}=0$ at the interface is loaded by a concentrated load $\mathbf{X}=-\left\{\tau_{0}, \sigma_{0}\right\}^{\mathrm{T}} \delta\left(x_{1}-d\right)$ at $x_{1}=d$ on its face, where $\tau_{0}$ and $\sigma_{0}$ are, respectively, the applied shear stress and normal stress. It is also assumed that the crack and load are extended with a speed $v$, less than the critical wave speed $c_{\text {cr }}$, in the $x_{1}$ direction and a frictionless contact zone $(a, b)$ of arbitrary length appears at the right of the crack tip. It has been shown that the longer contact zone develops at the right crack tip for the shear stress at infinity $\tau_{0}>0$ if the lower material is softer than the upper one and it develops for $\tau_{0}<0$ in the opposite case [34, 35]. Also it is demonstrated by Dundurs and Gautesen [36] and Kharun and Loboda [37] that neglecting the left short contact zone, the oscillating singularity at 
the left crack tip will not significantly influence the stress and strain fields at the right crack tip. Therefore, only the contact zone at the right crack tip is considered in the present study. The interface conditions in the moving coordinate system $\left(x_{1}, x_{3}\right)$ can be written as:

$$
\begin{aligned}
& {\left[\mathbf{U}\left(x_{1}\right)\right]=\mathbf{0},\left[\mathbf{t}\left(x_{1}\right)\right]=\mathbf{0}, x_{1} \notin(c, b)} \\
& \left\{\begin{array}{l}
\sigma_{13}\left(x_{1}, 0\right)=\tau_{0} \delta\left(x_{1}-d\right), \sigma_{33}\left(x_{1}, 0\right)=\sigma_{0} \delta\left(x_{1}-d\right), \\
{\left[\varphi\left(x_{1}\right)\right]=0,\left[D\left(x_{1}\right)\right]=0,\left[\phi\left(x_{1}\right)\right]=0,\left[B\left(x_{1}\right)\right]=0,}
\end{array} \quad x_{1} \in(c, a)\right. \\
& \left\{\begin{array}{l}
\sigma_{13}\left(x_{1}, 0\right)=0,\left[\sigma_{33}\left(x_{1}\right)\right]=0,\left[u_{3}\left(x_{1}\right)\right]=0, \\
{\left[\varphi\left(x_{1}\right)\right]=0,\left[D\left(x_{1}\right)\right]=0,\left[\phi\left(x_{1}\right)\right]=0,\left[B\left(x_{1}\right)\right]=0,}
\end{array}\right.
\end{aligned}
$$

where $\left[\Upsilon\left(x_{1}\right)\right]=\Upsilon^{+}\left(x_{1}, 0\right)-\Upsilon^{-}\left(x_{1}, 0\right)$ means the jump of function $\Upsilon\left(x_{1}\right)$ across the material interface; and“+” and “-” stand for the upper and lower surfaces of the crack, respectively.

By substituting Eqs.(12) and (14) into the boundary conditions Eqs.(15)-(17), one obtains an inhomogeneous combined Dirichlet-Riemann problem:

$$
\begin{aligned}
& \Omega_{1}^{+}\left(x_{1}\right)+\gamma_{1} \Omega_{j}^{-}\left(x_{1}\right)=t_{1}^{-1}\left(\sigma_{0}+\mathrm{i} m_{1} \tau_{0}\right) \delta\left(x_{1}-d\right), x_{1} \in(c, a) \\
& \operatorname{Im} \Omega_{1}^{ \pm}\left(x_{1}\right)=0, x_{1} \in(a, b) \\
& \left.\Omega_{1}(z)\right|_{z \rightarrow \infty}=0
\end{aligned}
$$

where "Im” stands for the imaginary part of the complex quantity.

The solution of the aforementioned problem defined by Eqs. (18)-(20) can be presented as [38]

$$
\Omega_{1}(z)=\frac{X(z)}{d-z}\left\{\operatorname{Re}\left(I_{0}\right)+\mathrm{i} \operatorname{Im}\left(I_{0}\right) \frac{Y(z)}{Y(d)}\right\}
$$

where

$$
X(z)=\frac{e^{\mathrm{i} \omega(z)}}{\sqrt{(z-c)(z-a)}}, I_{0}=\frac{1}{2 \pi \mathrm{i}} \frac{\sigma_{0}+\mathrm{i} m_{1} \tau_{0}}{t_{1} X^{+}(d)}, Y(z)=\sqrt{\frac{z-a}{z-b}}
$$


$\varpi(z)=2 \varepsilon_{1} \ln \frac{\sqrt{(b-a)(z-c)}}{\sqrt{l(z-a)}+\sqrt{(a-c)(z-b)}}, \varepsilon_{1}=\frac{1}{2 \pi} \ln \gamma_{1}, l=b-c$

Using the solution and Eq.(14), for $x_{1} \in(c, a)$, one obtains:

$$
\left[u_{1}^{\prime}\left(x_{1}\right)\right]+\mathrm{is}_{j}\left[u_{3}^{\prime}\left(x_{1}\right)\right]=\frac{\gamma_{1}+1}{\gamma_{1}} \frac{X\left(x_{1}\right)}{d-x_{1}}\left\{\operatorname{Re}\left(I_{0}\right)+\mathrm{i} \operatorname{Im}\left(I_{0}\right) \frac{Y\left(x_{1}\right)}{Y(d)}\right\}, x_{1} \in(c, a)
$$

Similarly, combining Eq. (12) with Eq. (20) leads to the following expressions for $x_{1} \in(a, b)$ :

$$
\begin{aligned}
\sigma_{33}^{(1)}\left(x_{1}, 0\right)= & -\frac{\chi_{1} \operatorname{Re}\left(I_{0}\right)}{\left(x_{1}-d\right) \sqrt{\left(x_{1}-c\right)\left(x_{1}-a\right)}}\left\{\cosh \varpi_{0}\left(x_{1}\right)+\frac{1-\gamma_{1}}{1+\gamma_{1}} \sinh \varpi_{0}\left(x_{1}\right)\right\}- \\
& \frac{\chi_{1} \operatorname{Im}\left(I_{0}\right)}{\left(x_{1}-d\right) \sqrt{\left(x_{1}-c\right)\left(x_{1}-a\right)}}\left\{\sinh \varpi_{0}\left(x_{1}\right)+\frac{1-\gamma_{1}}{1+\gamma_{1}} \cos \varpi_{0}\left(x_{1}\right)\right\} \frac{Y_{1}\left(x_{1}\right)}{Y(d)} \\
{\left[u_{1}^{\prime}\left(x_{1}\right)\right]=- } & \frac{2\left\{\operatorname{Re}\left(I_{0}\right) \sinh \varpi_{0}\left(x_{1}\right) Y(d)-Y_{1}\left(x_{1}\right) \operatorname{Im}\left(I_{0}\right) \cos \varpi_{0}\left(x_{1}\right)\right\}}{\left(x_{1}-d\right) \sqrt{\left(x_{1}-a\right)\left(x_{1}-c\right)} Y(d)}
\end{aligned}
$$

For $x_{1}>b$, one has

$$
\sigma_{33}^{(1)}\left(x_{1}, 0\right)+\mathrm{i} m_{1} \sigma_{13}^{(1)}\left(x_{1}, 0\right)=\chi_{1} \frac{X\left(x_{1}\right)}{d-x_{1}}\left\{\operatorname{Re}\left(I_{0}\right)+\mathrm{i} \operatorname{Im}\left(I_{0}\right) \frac{Y\left(x_{1}\right)}{Y(d)}\right\}, x_{1}>b
$$

where

$$
\varpi_{0}\left(x_{1}\right)=2 \varepsilon_{1} \tan ^{-1} \sqrt{\frac{(a-c)\left(b-x_{1}\right)}{(b-c)\left(x_{1}-a\right)}}, Y_{1}\left(x_{1}\right)=\sqrt{\frac{x_{1}-a}{b-x_{1}}}, \chi_{j}=t_{j}\left(1+\gamma_{j}\right)
$$

By means of Eqs. (8) and (12), the electric displacement and magnetic induction can be written as:

$$
\begin{aligned}
& D_{3}^{(1)}\left(x_{1}, 0\right)=\operatorname{Re}\left(g_{41}\right)\left[u_{1}^{\prime}\left(x_{1}\right)\right]+\frac{\operatorname{Im}\left(g_{41}\right) \operatorname{Im}\left(g_{13}\right)-\operatorname{Im}\left(g_{43}\right) g_{11}}{\left\{\operatorname{Im}\left(g_{31}\right)\right\}^{2}-g_{33} g_{11}}\left\{\sigma_{33}^{(1)}\left(x_{1}, 0\right)-\operatorname{Re}\left(g_{31}\right)\left[u_{1}^{\prime}\left(x_{1}\right)\right]\right\} \\
& B_{3}^{(1)}\left(x_{1}, 0\right)=\operatorname{Re}\left(g_{51}\right)\left[u_{1}^{\prime}\left(x_{1}\right)\right]+\frac{\operatorname{Im}\left(g_{51}\right) \operatorname{Im}\left(g_{13}\right)-\operatorname{Im}\left(g_{53}\right) g_{11}}{\left\{\operatorname{Im}\left(g_{31}\right)\right\}^{2}-g_{33} g_{11}}\left\{\sigma_{33}^{(1)}\left(x_{1}, 0\right)-\operatorname{Re}\left(g_{31}\right)\left[u_{1}^{\prime}\left(x_{1}\right)\right]\right\}
\end{aligned}
$$


Introducing the following FIFs,

$$
\begin{aligned}
& K_{1}=\lim _{x_{1} \rightarrow a+0} \sqrt{2 \pi\left(x_{1}-a\right)} \sigma_{33}^{(1)}\left(x_{1}, 0\right), K_{2}=\lim _{x_{1} \rightarrow b+0} \sqrt{2 \pi\left(x_{1}-b\right)} \sigma_{13}^{(1)}\left(x_{1}, 0\right) \\
& K_{D}=\lim _{x_{1} \rightarrow a+0} \sqrt{2 \pi\left(x_{1}-a\right)} D_{3}^{(1)}\left(x_{1}, 0\right), K_{B}=\lim _{x_{1} \rightarrow a+0} \sqrt{2 \pi\left(x_{1}-a\right)} B_{3}^{(1)}\left(x_{1}, 0\right)
\end{aligned}
$$

and using Eqs. (23) and (25), one arrives at the following expressions for $K_{1}$ and $K_{2}$

$$
\begin{aligned}
& K_{1}=-\sqrt{\frac{2}{\pi}} \frac{\sqrt{d-c}}{\sqrt{(a-d)(a-c)}}\left\{\hat{\sigma} \cos \varpi^{*}(d)+\hat{\tau} \sin \varpi^{*}(d)\right\} \\
& K_{2}=-\frac{1+\gamma_{1}}{\operatorname{Re}\left(m_{1}\right) \sqrt{2 \pi \gamma_{1}}} \frac{\sqrt{1-\theta}}{\sqrt{b-d}}\left\{\hat{\tau} \cos \varpi^{*}(d)-\hat{\sigma} \sin \varpi^{*}(d)\right\}
\end{aligned}
$$

where

$$
\begin{aligned}
& \hat{\sigma}=\sigma_{0}-\operatorname{Im}\left(m_{1}\right) \tau_{0}, \quad \hat{\tau}=\operatorname{Re}\left(m_{1}\right) \tau_{0} \\
& \varpi^{*}\left(x_{1}\right)=2 \varepsilon_{1} \ln \frac{\sqrt{(b-a)\left(x_{1}-c\right)}}{\sqrt{(b-c)\left(a-x_{1}\right)}+\sqrt{(a-c)\left(b-x_{1}\right)}}, \theta=\frac{b-d}{b-c}
\end{aligned}
$$

By using Eq. (27), $K_{D}$ and $K_{B}$ can be defined with $K_{1}$ and $K_{2}$, and presented as:

$$
\begin{aligned}
& K_{D}=\left\{\frac{\operatorname{Im}\left(g_{43}\right) g_{11}-\operatorname{Im}\left(g_{41}\right) \operatorname{Im}\left(g_{13}\right)}{g_{33} g_{11}-\left[\operatorname{Im}\left(g_{13}\right)\right]^{2}}+\left[\operatorname{Re}\left(g_{41}\right)+\frac{\operatorname{Re}\left(g_{31}\right)\left[\operatorname{Im}\left(g_{41}\right) \operatorname{Im}\left(g_{13}\right)-\operatorname{Im}\left(g_{43}\right) g_{11}\right]}{g_{33} g_{11}-\left[\operatorname{Im}\left(g_{13}\right)\right]^{2}}\right] \frac{\gamma_{1}^{2}-1}{2 \gamma_{1} \chi_{1}}\right\} K_{1} \\
& K_{B}=\left\{\frac{\operatorname{Im}\left(g_{53}\right) g_{11}-\operatorname{Im}\left(g_{51}\right) \operatorname{Im}\left(g_{13}\right)}{g_{33} g_{11}-\left[\operatorname{Im}\left(g_{13}\right)\right]^{2}}+\left[\operatorname{Re}\left(g_{51}\right)+\frac{\operatorname{Re}\left(g_{31}\right)\left[\operatorname{Im}\left(g_{51}\right) \operatorname{Im}\left(g_{13}\right)-\operatorname{Im}\left(g_{53}\right) g_{11}\right]}{g_{33} g_{11}-\left[\operatorname{Im}\left(g_{13}\right)\right]^{2}}\right] \frac{\gamma_{1}^{2}-1}{2 \gamma_{1} \chi_{1}}\right\} K_{1}
\end{aligned}
$$

The obtained equations are mathematically correct for any position of the point $a$, however, the solution only becomes physically valid if the following inequalities are satisfied:

$$
\begin{cases}\sigma_{33}^{(1)}\left(x_{1}, 0\right) \leq 0, & x_{1} \in(a, b) \\ {\left[u_{3}\left(x_{1}, 0\right)\right] \geq 0,} & x_{1} \in(c, a)\end{cases}
$$


It is shown that it is necessary and sufficient to satisfy the above inequalities and achieve a smooth closure of the crack contact zone if

$$
\lim _{x_{1} \rightarrow a-0} \sqrt{a-x_{1}}\left[u_{3}^{\prime}\left(x_{1}, 0\right)\right]=0
$$

holds true [31], which is equivalent to $K_{1}=0$ herein. Therefore, the length of the contact zone can be determined by using the following single transcendental equation:

$$
\tan \kappa(\lambda)=-\frac{\hat{\sigma}}{\hat{\tau}}
$$

where

$$
\kappa(\lambda)=2 \varepsilon_{1} \ln \frac{\sqrt{\lambda(1-\theta)}}{\sqrt{\theta-\lambda}+\sqrt{\theta(1-\lambda)}}, \lambda=\frac{b-a}{b-c}
$$

and $\lambda$ is the relative length of the contact zone.

It has been shown by Herrmann et al. [31] that the maximum root of Eq. (34) in the interval $(0,1)$ should be chosen if inequalities are satisfied (32). A small value of $\lambda(\lambda<<1)$ can be approximately written as:

$$
\lambda \approx \lambda_{0}=\frac{4 \theta}{1-\theta} \exp \left\{\frac{1}{\varepsilon_{1}}\left[\tan ^{-1}\left(-\frac{\hat{\sigma}}{\hat{\tau}}\right)+\pi n\right]\right\}
$$

and an appropriate value of $n$ should be chosen for the maximum value of $\lambda$.

Using Eq. (34), the stress intensity factor $K_{2}$ which corresponds to the real length of the contact zone is:

$$
K_{2}=-\frac{1}{\operatorname{Re}\left(m_{1}\right)} \frac{\left(1+\gamma_{1}\right) \sqrt{1-\theta}}{\sqrt{2 \pi \gamma_{1}(b-d)}} \Theta
$$


where $\Theta=\sqrt{\hat{\sigma}^{2}+\hat{\tau}^{2}}$. It should be noted that $K_{2}$ is the main fracture parameter of the crack model in this study. When the speed of the moving crack is zero and the poling direction of the lower material is along $x_{3}$-direction, the theoretical results in this case will reduce to those for static case presented by Herrmann et al. [27] and Feng et al. [28].

\section{Moving crack of semi-infinite length at interface under concentrated load}

The modeling of a moving crack of a finite length considered in the previous section was proposed by Yoffe [15] for a crack in a homogeneous material, and is suitable for examining local processes at the crack tip. However, together with this model, it is of interest to analyze a propagating crack of a semi-infinite length which was proposed by Goldshtein [39] for a case of two dissimilar isotropic materials. The results for this case can be obtained by using the above solution, in which the left of the crack tip $c$ tends to $-\infty$, i.e., $c \rightarrow-\infty$, as shown in Fig. 1b. In this situation, $X(z)$ and $I_{0}$ in Eq. (21a) become [38]:

$$
\left.X(z)\right|_{c \rightarrow \infty}=X_{\infty}(z)=\frac{e^{\mathrm{i} \omega_{\infty}(z)}}{\mathrm{i} \sqrt{\mathrm{z}-a}}, \quad I_{0}=\frac{\sqrt{d-a}}{2 \pi t_{1}} \Theta e^{\mathrm{i}\left[-\eta-\varpi_{\infty}(d)\right]}
$$

where

$$
\varpi_{\infty}(z)=-2 \varepsilon_{1} \ln \left(\sqrt{\frac{z-b}{b-a}}+\sqrt{1+\frac{z-b}{b-a}}\right), \quad \eta=-\tan ^{-1} \frac{\operatorname{Re}\left(m_{1}\right) \tau_{0}}{\sigma_{0}-\operatorname{Im}\left(m_{1}\right) \tau_{0}}
$$

Function $\Omega_{1}$ in Eq. (20) can be written in the form:

$$
\Omega_{1 \infty}=-\frac{\Theta e^{\mathrm{i} \omega_{\infty}(z)}}{2 \pi t_{1}(d-z)}\left\{\sin \left[\eta+\varpi_{\infty}(d)\right] \sqrt{\frac{d-b}{z-b}}+\mathrm{i} \cos \left[\eta+\varpi_{\infty}(d)\right] \sqrt{\frac{d-a}{z-a}}\right\}
$$

For convenience, the crack tip $b$ will be tied to the origin of the moving coordinate system $\left(x_{1}, x_{3}\right)$. Moreover, the length of the contact zone is denoted as $r$, and the distance from the 
applied concentrated load to the crack tip as $d_{1}$. It is clear that in this coordinate system, $b=0$, $a=-r$ and $d=-d_{1}$ hold true. Corresponding to the analysis of Eqs. (22)-(25), the following equations can be obtained in this case [31]

for $x_{1}<-r$,

$$
\left[u_{1}^{\prime}\left(x_{1}\right)\right]+\mathrm{i} s_{j}\left[u_{3}^{\prime}\left(x_{1}\right)\right]=t_{1} \Theta \frac{\gamma_{1}+1}{\gamma_{1}} \frac{\exp \left\{\mathrm{i} \varpi_{1}\left(x_{1}\right)\right\}}{2 \pi\left(x_{1}+d_{1}\right)}\left\{\sin (\eta+\vartheta) \sqrt{\frac{d_{1}}{-x_{1}}}+\mathrm{i} \cos (\eta+\vartheta) \sqrt{\frac{d_{1}-r}{-x_{1}-r}}\right\}
$$

and for $x_{1} \in(-r, 0)$,

$$
\begin{aligned}
& \sigma_{33}^{(1)}\left(x_{1}, 0\right)=-\frac{\Theta}{\pi\left(x_{1}+d_{1}\right)}\left\{\sin (\eta+\vartheta) \sinh \varpi_{1}\left(x_{1}\right) \sqrt{\frac{d_{1}}{-x_{1}}}+\cos (\eta+\vartheta) \cosh \varpi_{1}\left(x_{1}\right) \sqrt{\frac{d_{1}-r}{x_{1}+r}}\right\} \\
& {\left[u_{1}^{\prime}\left(x_{1}\right)\right]=\frac{\Theta}{\pi t_{1} \sqrt{\gamma_{1}}\left(x_{1}+d_{1}\right)} \sin (\eta+\vartheta) \sqrt{\frac{d_{1}}{-x_{1}}}, x_{1} \in(-r, 0)}
\end{aligned}
$$

For $x_{1}>0$

$$
\sigma_{33}^{(1)}\left(x_{1}, 0\right)+\mathrm{im}_{1} \sigma_{13}^{(1)}\left(x_{1}, 0\right)=\frac{\Theta\left(1+\gamma_{1}\right) \exp \left\{\mathrm{i} \varpi_{2}\left(x_{1}\right)\right\}}{2 \pi\left(x_{1}+d_{1}\right)}\left\{-\cos (\eta+\vartheta) \sqrt{\frac{d_{1}-r}{x_{1}+r}}+\mathrm{i} \sin (\eta+\vartheta) \sqrt{\frac{d_{1}}{x_{1}}}\right\}
$$

where

$$
\vartheta=-2 \varepsilon_{1} \ln \left(\sqrt{\frac{d_{1}}{r}-1}+\sqrt{\frac{d_{1}}{r}}\right), \varpi_{1}\left(x_{1}\right)=2 \varepsilon_{1} \tan ^{-1} \sqrt{\frac{r+x_{1}}{-x_{1}}}, \varpi_{2}(z)=-2 \varepsilon_{1} \ln \frac{1}{\mathrm{i}}\left(\sqrt{\frac{z}{r}}+\sqrt{1+\frac{z}{r}}\right)
$$

It is worth mentioning that Eq. (27) is still valid for electric displacement and magnetic induction in this case.

The FIFs in this situation can be obtained from Eq. (29) and presented as:

$$
\begin{aligned}
K_{1 \infty} & =-\sqrt{\frac{2}{\pi}} \frac{\Theta}{\sqrt{d_{1}-r}} \cos (\eta+\vartheta) \\
K_{2 \infty} & =-\frac{\Theta\left(1+\gamma_{1}\right)}{\operatorname{Re}\left(m_{1}\right) \sqrt{2 \pi d_{1} \gamma_{1}}} \sin (\eta+\vartheta)
\end{aligned}
$$


The equation for the length of the contact zone can be simplified as:

$\cos (\eta+\vartheta)=0$

It is convenient to introduce the parameters $\alpha=\lambda / \theta$ and $\alpha_{\infty}=r / d_{1}$ which define the coefficients of the length of the contact zone and the distance from the point load to the crack tip for cracks of finite and semi-infinite lengths, respectively. Therefore, the exact solution of Eq. (47) can be written as:

$\alpha_{\infty}=\cosh ^{-2}\left\{\frac{1}{2 \varepsilon_{1}}(\eta+\pi(0.5-n))\right\}$

and $n$ should be taken to be similar to that of the previous case. For a small value of $\alpha_{\infty}$, Eq. (49) can be approximately expressed as:

$\alpha_{\infty} \approx \tilde{\alpha}_{\infty}=4 \exp \left\{-\frac{1}{\varepsilon_{1}}(\eta+\pi(0.5-n))\right\}$

By using Eq. (47), the stress intensity factor $K_{2}$ for a crack of semi-infinite length at the interface

can be expressed as:

$K_{2 \infty}=-\frac{\Theta\left(1+\gamma_{1}\right)}{\operatorname{Re}\left(m_{1}\right) \sqrt{2 \pi d_{1} \gamma_{1}}}$

A comparison between Eqs. (37) and (50) shows that:

$\tilde{\alpha}_{\infty}=(1-\theta) \tilde{\lambda}_{0} / \theta, K_{2 \infty}=K_{2} /(1-\theta)$.

\section{Moving crack of finite length at interface under remote mixed-mode loading}

Consider a moving crack of a finite length at the interface, as shown in Fig. 2, under remote mixmode loading $\sigma_{33}=\sigma_{0}, \sigma_{13}=\tau_{0}$. As previously mentioned in Section 4, crack surfaces are traction-free for $x_{1} \in(c, a)$ but have frictionless contact for $x_{1} \in(a, b)$. The solution of the 
problem considered can be constructed as the sum of two parts-a state of uniform loading $\left(\tau_{0}, \sigma_{0}\right)$, and a perturbed field caused by ME permeable crack faces that tend to zero at infinity.

Since the homogeneous field is beyond the scope of this study, we consider the perturbed state with continuity and boundary conditions at the interface in the following form:

$$
\begin{aligned}
& {\left[\mathbf{U}\left(x_{1}\right)\right]=\mathbf{0},\left[\mathbf{t}\left(x_{1}\right)\right]=\mathbf{0}, x_{1} \notin(c, b)} \\
& \left\{\begin{array}{l}
\sigma_{13}\left(x_{1}, 0\right)=-\tau_{0}, \sigma_{33}\left(x_{1}, 0\right)=-\sigma_{0}, \\
{\left[\varphi\left(x_{1}\right)\right]=0,\left[D\left(x_{1}\right)\right]=0,\left[\phi\left(x_{1}\right)\right]=0,\left[B\left(x_{1}\right)\right]=0,}
\end{array} \quad x_{1} \in(c, a)\right. \\
& \left\{\begin{array}{l}
\sigma_{13}\left(x_{1}, 0\right)=0,\left[\sigma_{33}\left(x_{1}\right)\right]=0,\left[u_{3}\left(x_{1}\right)\right]=0, \\
{\left[\varphi\left(x_{1}\right)\right]=0,\left[D\left(x_{1}\right)\right]=0,\left[\phi\left(x_{1}\right)\right]=0,\left[B\left(x_{1}\right)\right]=0,}
\end{array} \quad x_{1} \in(a, b)\right.
\end{aligned}
$$

Moreover, as previously mentioned, $W_{4}\left(x_{1}\right)=W_{5}\left(x_{1}\right)=0$ still holds true since there is no loading at infinity in the perturbed field.

By performing an analogous derivation for Eqs. (18)-(20) and introducing a new function:

$\Phi_{j}(z)=\Omega_{j}(z)+\frac{\sigma_{0}+\mathrm{i} m_{j} \tau_{0}}{\chi_{j}}$

one obtains:

$$
\begin{aligned}
& \sigma_{33}^{(1)}\left(x_{1}, 0\right)+\mathrm{i} m_{j} \sigma_{13}^{(1)}\left(x_{1}, 0\right)=t_{j}\left\{\Phi_{j}^{+}\left(x_{1}\right)+\gamma_{j} \Phi_{j}^{-}\left(x_{1}\right)\right\}-\left(\sigma_{0}+\mathrm{i} m_{j} \tau_{0}\right) \\
& {\left[u_{1}^{\prime}\left(x_{1}\right)\right]+\mathrm{i} s_{j}\left[u_{3}^{\prime}\left(x_{1}\right)\right]=\Phi_{j}^{+}\left(x_{1}\right)-\Phi_{j}^{-}\left(x_{1}\right)} \\
& \left.\Phi_{j}(z)\right|_{z \rightarrow \infty}=\frac{\sigma_{0}+\mathrm{i} m_{j} \tau_{0}}{\chi_{j}}
\end{aligned}
$$

Using Eqs. (56)-(58) for $j=1$ and the corresponding interface conditions, one arrives at the following combined Dirichlet-Riemann boundary value problem:

$$
\Phi_{1}^{+}\left(x_{1}\right)+\gamma_{1} \Phi_{1}^{-}\left(x_{1}\right)=0, \quad x_{1} \in(c, a)
$$


$\operatorname{Im} \Phi_{1}^{ \pm}\left(x_{1}\right)=0, \quad x_{1} \in(a, b)$

$\left.\Phi_{1}(z)\right|_{z \rightarrow \infty}=\frac{\sigma_{0}+\mathrm{i} m_{1} \tau_{0}}{\chi_{1}}$

The exact analytical solution of Eqs. (59)-(61) was first provided by Herrmann and Loboda [33] and for brevity, it is omitted herein. Using Eqs. (55) and (57), one can obtain the following expressions at the interface

for $x_{1}>b$,

$$
\sigma_{33}^{(1)}\left(x_{1}, 0\right)+\mathrm{i} m_{1} \sigma_{13}^{(1)}\left(x_{1}, 0\right)=\left\{\frac{Q\left(x_{1}\right)}{\sqrt{x_{1}-a}}+\mathrm{i} \frac{P\left(x_{1}\right)}{\sqrt{x_{1}-b}}\right\} \frac{\chi_{1} \mathrm{e}^{\mathrm{i} \sigma\left(x_{1}\right)}}{\sqrt{x_{1}-c}}-\left(\sigma_{0}+\mathrm{i} m_{1} \tau_{0}\right)
$$

for $x_{1} \in(a, b)$,

$$
\begin{array}{r}
\sigma_{33}^{(1)}\left(x_{1}, 0\right)=\frac{\chi_{1} P\left(x_{1}\right)}{\sqrt{\left(x_{1}-c\right)\left(b-x_{1}\right)}}\left[\frac{1-\gamma_{1}}{1+\gamma_{1}} \cosh \varpi_{0}\left(x_{1}\right)+\sinh \varpi_{0}\left(x_{1}\right)\right] \\
+\frac{\chi_{1} Q\left(x_{1}\right)}{\sqrt{\left(x_{1}-c\right)\left(x_{1}-a\right)}}\left[\cosh \varpi_{0}\left(x_{1}\right)+\frac{1-\gamma_{1}}{1+\gamma_{1}} \sinh \varpi_{0}\left(x_{1}\right)\right]-\sigma_{0} \\
{\left[u_{1}^{\prime}\left(x_{1}\right)\right]=\frac{2}{\sqrt{x_{1}-c}}\left[\frac{P\left(x_{1}\right)}{\sqrt{b-x_{1}}} \cosh \varpi_{0}\left(x_{1}\right)+\frac{Q\left(x_{1}\right)}{\sqrt{x_{1}-a}} \sinh \varpi_{0}\left(x_{1}\right)\right]}
\end{array}
$$

for $x_{1} \in(c, a)$,

$$
\left[u_{1}^{\prime}\left(x_{1}\right)\right]+\mathrm{i} s_{j}\left[u_{3}^{\prime}\left(x_{1}\right)\right]=\frac{\gamma_{1}+1}{\sqrt{\gamma_{1}}}\left\{\frac{P\left(x_{1}\right)}{\sqrt{b-x_{1}}}-\mathrm{i} \frac{Q\left(x_{1}\right)}{\sqrt{a-x_{1}}}\right\} \frac{e^{\mathrm{i} \sigma^{*}\left(x_{1}\right)}}{\sqrt{x_{1}-c}}
$$

where $P(z)$ and $Q(z)$ can be found in the work by Herrmann and Loboda [33] with $\tilde{\sigma}_{1}, \tilde{\tau}_{1}$ as well as $\tilde{\sigma}_{4}, \tilde{\tau}_{4}$ later being defined by the formulas:

$$
\tilde{\sigma}_{j}=\frac{\hat{\sigma}}{\chi_{j}}, \tilde{\tau}_{j}=-\frac{\hat{\tau}}{\chi_{j}}, j=1,3
$$


and $\hat{\sigma}, \hat{\tau}$ can be found in Eq. (30).

According to the definition of FIFs in Eq. (28), one can obtain:

$$
\begin{aligned}
& K_{1}=\frac{\sqrt{2 \pi l \gamma_{1}}}{1+\gamma_{1}}\left\{\sqrt{1-\lambda}(\hat{\sigma} \cos \beta+\hat{\tau} \sin \beta)-2 \varepsilon_{1}(\hat{\sigma} \sin \beta-\hat{\tau} \cos \beta)\right\} \\
& K_{2}=-\frac{1}{m_{1}} \sqrt{\frac{\pi l}{2}}\left\{(\hat{\sigma} \sin \beta-\hat{\tau} \cos \beta)+2 \varepsilon_{1} \sqrt{1-\lambda}(\hat{\sigma} \cos \beta+\hat{\tau} \sin \beta)\right\}
\end{aligned}
$$

where $\beta=\varepsilon_{1} \ln \frac{1-\sqrt{1-\lambda}}{1+\sqrt{1-\lambda}}$. It should be noted that the expressions for the FIFs in Eq. (31) are still valid in this case.

Similarly, inequalities in Eq. (32) should be addressed to produce a real contact zone at the right of the crack tip. This leads to the following transcendental equation:

$$
\tan \beta=\frac{\sqrt{1-\lambda} \hat{\sigma}+2 \varepsilon_{1} \hat{\tau}}{2 \varepsilon_{1} \hat{\sigma}-\sqrt{1-\lambda} \hat{\tau}}
$$

For a small contact zone, the following asymptotic solution of Eq. (68) can be used:

$$
\lambda \approx \lambda_{0}=4 \exp \left\{-\frac{1}{\varepsilon}\left[\tan ^{-1}(2 \varepsilon)-\tan ^{-1} \frac{\hat{\sigma}}{\hat{\tau}}-\pi(n-0.5)\right]\right\}
$$

where $n$ which defines the maximum $\lambda_{0}$ in the interval $(0,1)$ should be taken.

Using Eq. (68), Eq. (67b) can be rewritten in the form of:

$$
K_{2}=-\frac{1}{m_{1}} \sqrt{\frac{\pi l}{2}}(\hat{\sigma} \sin \beta-\hat{\tau} \cos \beta)\left\{1+\left(2 \varepsilon_{1}\right)^{2}\right\}
$$

When the speed of the crack is zero and the poling direction of the lower material is along $x_{3}$ direction, the theoretical results will reduce to those for static case by Herrmann et al. [27].

\section{Numerical results and discussion}


This section provides the numerical results that are related to a moving crack under concentrated and uniform mix-mode loading at the interface, respectively. The upper and lower MEE materials are composites made of $\mathrm{BaTiO}_{3}$ as the inclusion material and $\mathrm{CoFe}_{2} \mathrm{O}_{4}$ as the matrix material. Their properties are shown in Table $1[40,41]$. The following mixture rule is used to determine the composite material constants:

$$
\Lambda^{(\mathrm{c})}=\Lambda^{(\mathrm{i})} V_{\mathrm{f}}+\Lambda^{(\mathrm{m})}\left(1-V_{\mathrm{f}}\right)
$$

where superscripts c, i, and $\mathrm{m}$ denote the composite, inclusion and matrix materials, respectively, and $V_{\mathrm{f}}$ is the volume fraction of the piezoelectric inclusion. The readers can also use other rules to determine the magnetoelectric coefficient from the point of view of micromechanics [42-44], which is more rigorous.

In the following figures, only the numerical results related to the crack of finite length at the interface are presented since the corresponding quantities in relation to the semi-infinite crack at the interface are dependent on the former case (see Eq.(51)). The relative length of the contact zone $\lambda$ for a finite crack is defined as $\lambda=(b-a) / l$, and $l=b-c$. As previously mentioned, the speed of a moving crack is assumed to be less than the minimum Rayleigh wave speed of a bimaterial system, which is the critical wave speed $v_{\text {cr }}$. Since an analytical expression for the Rayleigh wave speed of MEE materials is usually not available, the speed of a moving crack is normalized by using the speed of the shear wave $v_{0}$ of the $\mathrm{BaTiO}_{3}-\mathrm{CoFe}_{2} \mathrm{O}_{4}$ composite with a volume fraction of piezoelectric inclusion of $V_{\mathrm{f}}=0.1$ in all of the numerical results, where $v_{0}=\sqrt{\mu / \rho}$ and $\mu=c_{44}+\frac{\alpha_{11} h_{15}^{2}-2 e_{15} h_{15} d_{11}+\mu_{11} e_{15}^{2}}{\mu_{11} \alpha_{11}-d_{11}^{2}}[14,23]$. The normalized critical wave speed $v_{\mathrm{cr}} / v_{0}$ related to different material volume fractions and poling directions is listed in Table 2. 
Although the extended stresses are not oscillating at the right of the crack tip for the crack model in this study, the oscillating index, which is an important parameter in classical moving crack models, is also provided for reference herein. Only when the speed of the moving crack is less than $v_{\text {cr }}$ the oscillating index is a real number; otherwise, the oscillating index will become a complex number [45] and the solution procedure is entirely different from that in this work. In Fig. 3, the variation of the oscillating index $\varepsilon_{1}$ with respect to the speed of the moving crack for different poling directions and material volume fractions is presented, respectively, where $V_{\mathrm{f} 1}$ and $V_{\mathrm{f} 2}$ are the volume fraction of the piezoelectric inclusion of the upper and lower materials, respectively. For $V_{\mathrm{f} 1}=0.99$, the corresponding material is almost a piezoelectric material whereas for $V_{\mathrm{f} 2}=0.01$, almost a piezomagnetic material. The results shown in Fig. 3 show that the oscillating index $\varepsilon_{1}$ increases as the speed of the moving crack increases in all cases, and when the speed of the moving crack approaches the critical wave speed, $\varepsilon_{1}$ increases much more rapidly and tends to infinity, which is consistent with the observations made by Shen and Nishioka [32] and Shen et al. [45] in relation to anisotropic elastic and piezoelectric bimaterials, respectively. Additionally, the difference in the oscillating index $\varepsilon_{1}$ for different poling directions becomes obvious for cracks that move at a faster speed. There is only a slight difference in the oscillating index between $\beta_{0}=0$ and $\beta_{0}=\pi$ for a crack that moves at an arbitrary speed. Moreover, Fig. 3b demonstrates that increasing the differences in the properties between the upper and lower materials, which can be defined as the difference between $V_{\mathrm{f} 1}$ and $V_{\mathrm{f} 2}$, leads to an increase in $\varepsilon_{1}$. This agrees with our expectations. 
It should be noted that a contact zone does not always exist for cracks that move at an arbitrary rate and arbitrary poling direction since the prerequisite defined by inequality (32) should be satisfied. Thus only some typical numerical results are presented in this section. Figs. 4 and 5 show the dependence of the relative length of the contact zone $\lambda$ and the normalized FIF $K_{2} / K_{0}$

of a finite crack on the normalized speed of a moving crack $v / v_{0}$ for different poling directions and material volume fractions under concentrated loading, where the load ratio is $k=\tau_{0} / \sigma_{0}$ and $K_{0}=\sigma_{0} / \sqrt{0.5 l}$ respectively. Under pure tensile loading, i.e. $k=0$, the length of the contact zone is very small; therefore, it is given at a logarithmic scale. In Figs. 4 and 5, it is shown that an increase in the speed of a moving crack always leads to monotone increases in the relative length of the contact zone $\lambda$ and the normalized FIF $K_{2} / K_{0}$ for $k=0$ in all cases. As the speed of moving approaches a critical speed, the relative length of the contact zone $\lambda$ and the normalized FIF $K_{2} / K_{0}$ increases much more rapidly, and the former may be larger than 0.25 even for $k=0$, and thus the introduction of a contact zone is very necessary for this problem. All these are consistent with the observations made by Herrmann et al. [31] for piezoelectric bimaterials. As expected, the relative length of the contact zone and the FIF increase as the difference in material properties between the upper and lower MEE materials increase, which can be defined by the differences between $V_{\mathrm{f} 1}$ and $V_{\mathrm{f} 2}$ in Figs. $4 \mathrm{~b}$ and 5b. The bimaterial composed of a piezoelectric material $\left(V_{\mathrm{f} 1}=0.99\right)$ and a piezomagnetic material $\left(V_{\mathrm{f} 2}=0.01\right)$ produces the largest length of the contact zone. However, the relative length of the contact zone and the normalized FIF for $\beta_{0}=0$ and $\beta_{0}=\pi$ are very close. This is different from the findings in Herrmann et al. [31] for 
piezoelectric bimaterial composed of PZT-4 and PZT-5, but consistent with the observation under some circumstances by Sih et al. [46] for a homogeneous MEE solid. Therefore, it can be attributed to the special material properties of MEE bimaterials, where the piezomagnetic phase is further introduced and the coupling among elastic, electric and magnetic fields is achieved.

In the work by Herrmann et al. [31], the influence of the speed of the moving crack on the fracture parameters under combined tension-shear loading was not investigated. Therefore, this is further examined in this study with the numerical results plotted in Figs. 6 and 7. It can be seen in Fig. 6 that when a moderate shear load is applied, i.e. load ratio $k=5$, the length of the contact zone still monotonically increases as the speed of the moving crack increases for all poling directions and material volume fractions. For a specified poling direction, the relative length of the contact zone for $k=5$ is always much larger than that for $k=0$ in Fig. 4a. Additionally, Fig. 6a demonstrates that the relative length of the contact zone for $\beta_{0}=\pi$ is larger than that for $\beta_{0}=0$. This is opposite to the results shown in Fig. 4a. Also, the FIF for $\beta_{0}=0$ is larger than that for $\beta_{0}=\pi / 3$ in Fig. 7a whilst the former is smaller than the latter in Fig. 5a. These imply that the conclusions in relation to pure tensile loading may be different from those in relation to combined tensile-shear loading owing to the interaction of the presence of shear load and the variations in the poling direction and the speed of the moving crack. The results shown in Figs. $6 \mathrm{~b}$ and $7 \mathrm{~b}$ show that reducing the difference between $V_{\mathrm{f} 1}$ and $V_{\mathrm{f} 2}$ leads to reductions in the relative length of the contact zone and the FIF, which is analogous to the results plotted in Figs. $4 \mathrm{~b}$ and $5 \mathrm{~b}$.

Figs. 8 and 9 show the relationship between the fracture parameters of a finite crack and speed of the moving crack under different load ratios $k$ and load positions $\theta$ under concentrated loading. 
It can be observed that for a moderate shear load, the relative length of the contact zone and the FIF increase with increases in the normalized speed of the moving crack and load ratio $k$. An increase in load position $\theta$ results in an increase in length of the contact zone and a decrease of the FIF. These conclusions fully agree with the static fracture analysis of interface cracks in MEE bimaterials [27, 28]. Additionally, a comparison between Figs. 7b and 9 shows that compared to the poling direction and material volume fraction, load position $\theta$ and load ratio $k$ have a more significant influence on the normalized FIF $K_{2} / K_{0}$.

The variations in the relative length of the contact zone and the normalized FIF of a finite crack with respect to the normalized speed of the moving crack under uniform mixed-mode loading are presented in Figs. 10 and 11, respectively. The corresponding conclusions are similar to the previous ones. For moderate combined tension-shear loading, an increase in the difference between $V_{\mathrm{f} 1}$ and $V_{\mathrm{f} 2}$ leads to increases in the relative length of the contact zone and the normalized FIF. Additionally, the fracture parameters for $\beta_{0}=0$ and $\pi$ are also very close. It is remarked that when the speed of the moving crack is zero, the extended displacement discontinuity boundary element method (EDDBEM) proposed by Zhao et al. [47], which is an efficient approach for the analysis of fracture problems, can also be used to deal with the present problem.

\section{Conclusions}

A moving crack with a frictionless contact zone at the interface of MEE bimaterial is taken into consideration. The speed of the moving crack is assumed to be less than that of the critical shear wave of the bimaterial system. Three cases, i.e. a finite crack and a semi-infinite crack under concentrated loading, and a finite crack under remote mix-mode loading, are studied, respectively. 
By introducing a moving coordinate system and using boundary conditions, a combined Dirichlet-Riemann problem is formulated and solved analytically for each case. The transcendental equation for determining the length of the contact zone and FIF is further derived. Finally, numerical results are provided graphically for the material combination of $\mathrm{BaTiO}_{3}$ $\mathrm{CoFe}_{2} \mathrm{O}_{4}$ composites. The effects of the speed of the moving crack, poling direction, load ratio, load position and material volume fraction on the length of the contact zone and FIF are investigated in detail. The following conclusions are drawn from the numerical results.

(i) An increase in the speed of a moving crack causes an increase in the oscillating index.

(ii) In the crack model in this study, increases in the speed of the moving crack and shear loading usually lead to increases in the relative length of the contact zone and the FIF $K_{2}$ under both concentrated and mix-mode loadings.

(iii)For $\mathrm{BaTiO}_{3}-\mathrm{CoFe}_{2} \mathrm{O}_{4}$ composites, an increase in the difference of the material properties between the upper and the lower materials, which can be defined by the difference in material volume fraction, usually leads to increases in the length of the contact zone and the FIF $K_{2}$.

(iv)Compared with the material volume fraction and poling direction, load position and load ratio have more significant effects on the FIF $K_{2}$ when a concentrated load is applied onto the crack face. 


\section{Acknowledgement}

Support from the General Research Fund of Hong Kong (HKU 17223916), the National Natural Science Foundation of China (Grant Nos. 11572358, 10772123 and 11072160) and the Training Program for Leading Talent in University Innovative Research Team in Hebei Province (LJRC006) is gratefully acknowledged.

\section{References}

[1] Gao CF, Noda N. Thermal-induced interfacial cracking of magnetoelectroelastic materials. Int J Eng Sci 2004;42: 1347-1360.

[2] Li R, Kardomateas GA. The mixed Mode I and II interface crack in piezoelectromagnetoelastic anisotropic bimaterials. J Appl Mech 2007;74: 614-627.

[3] Huang GY, Wang BL, Mai YW. Effect of interfacial cracks on the effective properties of magnetoelectroelastic composites. J Intel Mater Syst Struct 2009;20: 963-968.

[4] Feng WJ, Su RKL, Liu JX, Li YS. Fracture analysis of bounded magnetoelectroelastic layers with interfacial cracks under magnetoelectromechanical loads: plane problem. J Intel Mater Syst Struct 2010;21: 581-594.

[5] Ma P, Su RKL, Feng WJ. Integral identities based on symmetric and skew-symmetric weight functions for a semi-infinite interfacial crack in anisotropic magnetoelectroelastic bimaterials. Int J Solids Struct 2016;88-89: 178-191.

[6] Feng WJ, Xue Y, Zou ZZ. Crack growth of an interface crack between two dissimilar magneto-electro-elastic materials under anti-Plane mechanical and in-plane electric magnetic impact. Theor Appl Fract Mech 2005;43: 376-394.

[7] Feng WJ, Pan E. Dynamic fracture behavior of an internal interfacial crack between two dissimilar magneto-electro-elastic plates. Eng Fract Mech 2008;75: 1468-1487. 
[8] Peng XL, Li XF. Transient response of the crack-tip field in a magnetoelectroelastic halfSpace with a functionally graded coating under impacts. Arch Appl Mech 2009;79: 10991113.

[9] Wang BL, Han JC, Du SY. Transient fracture of a layered magnetoelectroelastic medium. Mech Mater 2010;42: 354-364.

[10] $\mathrm{Hu} \mathrm{K}$, Chen Z, Fu J. Dynamic analysis of an interface crack between magnetoelectroelastic and functionally graded elastic layers under anti-plane mechanical and in-plane electromagnetic loadings. Compos Struct 2014;107: 142-148.

[11] Zhong XC, Liu F, Li XF. Transient response of a magnetoelectroelastic solid with two collinear dielectric cracks under impacts. Int J Solids Struct 2009;46: 2950-2958.

[12] Chen X. Energy release rate and path-independent integral in dynamic fracture of magnetoelectro-thermo-elastic solids. Int J Solids Struct 2009;46: 2706-2711.

[13] Hu K, Chen Z. Pre-curving analysis of an opening crack in a magnetoelectroelastic strip under in-plane impact loadings. J Appl Phys 2012;112: 124911.

[14] Feng WJ, Li YS, Xu ZH. Transient response of an interfacial crack between dissimilar magnetoelectroelastic layers under magnetoelectromechanical impact loadings: Mode-I problem. Int J Solids Struct 2009;46: 3346-3356.

[15] Yoffe EH. The moving Griffith crack. Philos Mag 1951;42: 739-750.

[16] Willis JR. Fracture mechanics interfacial cracks. J Mech Phys Solids 1971;19: 353-368.

[17] Atkinson C. Dynamic crack problems in dissimilar media. Mech Fract, Noordhoff International Publishing, Leyden, 213-248.

[18] Hu KQ, Li GQ. Constant moving crack in a magnetoelectroelastic material under anti-plane shear loading. Int J Solids Struct 2005;42: 2823-2835. 
[19] Hu KQ, Kang YL, Qin QH. A moving crack in a rectangular magnetoelectroelastic body. Eng Fract Mech 2007;74: 751-770.

[20] $\mathrm{Fu} \mathrm{J}$, Hu K, Chen $\mathrm{Z}$, et al.. A moving crack propagating in a functionally graded magnetoelectroelastic strip under different crack face conditions. Theor Appl Fract Mech 2013;66: 16-25.

[21] Tupholme GE. Magnetoelectroelastic media containing a row of moving shear cracks. Mech Res Commun 2012;45: 48-53.

[22] Hu K, Chen Z. Pre-Kinking of a moving crack in a magnetoelectroelastic material under inplane loading. Int J Solids Struct 2013;50: 2667-2677.

[23] Hu K, Chen Z, Zhong Z. Pre-kinking analysis of a constant moving crack in a magnetoelectroelastic strip under in-plane loading. Eur J Mech A-Solid 2014;43: 25-43.

[24] Zhong XC, Li XF. A finite length crack propagating along the interface of two dissimilar magnetoelectroelastic materials. Int J Eng Sci 2006;44: 1394-1407.

[25] Chen HS, Wei WY, Liu JX, Fang DN. Propagation of a Mode-III interfacial crack in a piezoelectric-piezomagnetic bi-material. Int J Solids Struct 2012;49: 2547-2558.

[26] Hu K, Chen Z, Fu J. Moving Dugdale crack along the interface of two dissimilar magnetoelectroelastic materials. Acta Mech 2015;226: 2065-2076.

[27] Herrmann KP, Loboda V, Khodanen T. An interface crack with contact zones in a piezoelectric/piezomagnetic bimaterial. Arch Appl Mech 2010; 80: 651-670.

[28] Feng WJ, Ma P, Pan E, Liu JX. A magnetically impermeable and electrically permeable interface crack with a contact zone in a magnetoelectroelastic bimaterial under concentrated magnetoelectromechanical loads on the crack faces. Sci China: Phys, Mech Astron 2011;54: 1666-1679. 
[29] Feng WJ, Ma P, Su RKL. An electrically impermeable and magnetically permeable interface crack with a contact zone in magnetoelectroelastic bimaterials under a thermal flux and magnetoelectromechanical loads. Int J Solids Struct 2012; 49: 3472-3483.

[30] Huang JH, Kuo WS. The analysis of piezoelectric/piezomagnetic composite materials containing ellipsoidal inclusions. J Appl Phys 1997;81: 1378-1386.

[31] Herrmann KP, Komarov AV, Loboda VV. On a moving interface crack with a contact zone in a piezoelectric bimaterial. Int J Solids Struct 2005;42: 4555-4573.

[32] Shen S, Nishioka T. A unified method for subsonic and intersonic crack growth along an anisotropic bimaterial interface. J Mech Phys Solids 2000;48: 2257-2282.

[33] Herrmann KP, Loboda VV. Fracture mechanical assessment of electrically permeable interface cracks in piezoelectric bimaterials by consideration of various contact zone models. Arch Appl Mech 2000;70: 127-143.

[34] Loboda VV. Analytical derivation and investigation of the interface crack models. Int J Solids Struct 1998;35: 4477-4489.

[35] Herrmann KP, Loboda VV, Govorukha VB. On contact zone models for an electrically impermeable interface crack in a piezoelectric bimaterial. Int J Fract 2001;111: 203-227.

[36] Dundurs J, Gautesen AK. An opportunistic analysis of the interface crack. Int J Fract 1988;36: 151-159.

[37] Kharun IV, Loboda VV. A set of interface cracks with contact zones in combined tensionshear field. Acta Mech 2003;166: 43-56.

[38] Herrmann KP, Loboda VV, Komarov AV. Contact zone assessment for a fast growing interface crack in an anisotropic bimaterial. Arch Appl Mech 2004;74:118-129.

[39] Goldshtein RV. On the steady motion of a crack along a straight line boundary between two joined material. Mech Tverdogo Tela (in Russian) 1996;1: 94-102. 
[40] Huang JH, Chiu YH, Liu HK. Magneto-electro-elastic Eshelby tensors for a piezoelectricpiezomagnetic composite reinforced ellipsoidal inclusions. J Appl Phys 1998;83: 5364-5370.

[41] Pan E. Three-dimensional Green's functions in anisotropic magneto-electro-elastic bimaterials. Zeitschrift für angewandte Mathematik und Physik ZAMP 2002;53: 815-838.

[42] Li, JY, Dunn, ML. Micromechanics of magnetoelectroelastic composite materials: Average fields and effective behavior. J Intel Mater Syst Struct 1998; 9: 404-416.

[43] Pan E, Wang R. Effects of geometric size and mechanical boundary conditions on magnetoelectric coupling in multiferroic composites. J Phys D: Appl Phys 2009;245503.

[44] Sladek J, Sladek V, Pan E. Effective properties of coated fiber composites with piezoelectric and piezomagnetic phases. J Intel Mater Syst Struct 2017;28: 97-107.

[45] Shen S, Nishioka T, Hu SL. Crack propagation along the interface of piezoelectric bimaterial. Theor Appl Fract Mech 2000;34: 185-203.

[46] Sih GC, Jones R, Song ZF. Piezomagnetic and piezoelectric poling effects on mode I and II crack initiation behavior of magnetoelectroelastic materials. Theor Appl Fract Mech 2003;40: 161-186.

[47] Zhao M, Zhang Q, Pan E., Fan C. Fundamental solutions and numerical modeling of an elliptical crack with polarization saturation in a transversely isotropic piezoelectric medium. Eng Fract Mech 2014;131: 627-642. 


\section{Table and Figure Captions}

Table 1 Material properties of $\mathrm{BaTiO}_{3}$ and $\mathrm{CoFe}_{2} \mathrm{O}_{4}[40,41]$

Table 2 Normalized critical wave speed $v_{\text {cr }} / v_{0}$ for $\mathrm{BaTiO}_{3}-\mathrm{CoFe}_{2} \mathrm{O}_{4}$ composites with different poling directions and material volume fractions

Fig. 1. Moving crack with frictionless contact zone at interface of MEE bimaterial under concentrated loading: (a) finite crack, and (b) semi-infinite crack

Fig. 2. Moving crack with frictionless contact zone at interface of MEE bimaterial under uniform mixed-mode loading

Fig. 3. Variation of the oscillating index $\varepsilon_{1}$ with respect to normalized speed of the moving crack $v / v_{0}$ for different (a) poling directions, and (b) material volume fractions

Fig. 4. Relative length of contact zone $\lambda$ of finite crack with respect to normalized speed of moving crack $v / v_{0}$ for different (a) poling directions, and (b) material volume fractions under concentrated loading with $k=0$

Fig. 5. Normalized FIF $K_{2} / K_{0}$ of finite crack with respect to normalized speed of moving crack $v / v_{0}$ for different (a) poling directions, and (b) material volume fractions under concentrated loading with $k=0$

Fig. 6. Relative length of contact zone $\lambda$ of finite crack with respect to normalized speed of moving crack $v / v_{0}$ for different (a) poling directions, and (b) material volume fractions under concentrated loading with $k=0$ 
Fig. 7. Normalized FIF $K_{2} / K_{0}$ of finite crack with respect to normalized speed of moving crack $v / v_{0}$ for different (a) poling directions, and (b) material volume fractions under concentrated loading with $k=0$

Fig. 8. Relative length of contact zone $\lambda$ of finite crack with respect to normalized speed of moving crack $v / v_{0}$ for different load positions and load ratios under concentrated loading

Fig. 9. Normalized FIF $K_{2} / K_{0}$ with respect to normalized speed of moving crack $v / v_{0}$ for different load positions and load ratios under concentrated loading

Fig. 10. Relative length of contact zone $\lambda$ of finite crack with respect to normalized speed of moving crack $v / v_{0}$ for different load positions and load ratios under uniform mixed-mode loading

Fig. 11. Normalized FIF $K_{2} / K_{0}$ of finite crack with respect to normalized speed of moving crack $v / v_{0}$ for different load positions and load ratios under uniform mixed-mode loading 


\section{Nomenclature}

$\left(X_{1}, X_{2}, X_{3}\right)$ fixed coordinate system

$\left(x_{1}, x_{2}, x_{3}\right)$ moving coordinate system

$c_{i j k l}, e_{i k l}, h_{i k l}, d_{i l}$ elastic, piezoelectric, piezomagnetic, and electromagnetic constants

$\alpha_{i l}, \mu_{l i}$ dielectric permittivities and magnetic permeabilities

$\sigma_{i j}, D_{i}, B_{i}$ stresses, electric displacements and magnetic inductions

$u_{i}, \varphi, \phi$ mechanical displacements, electric potentials and magnetic potentials

$\rho$ material density

$v$ speed of the crack

$C_{\mathrm{cr}} \quad$ critical surface wave speed

$\beta_{0} \quad$ poling direction of the upper material

$\tau_{0}, \sigma_{0}$ applied shear stress and normal stress

$c, a, b \quad x$-coordinates of crack tips

$K_{1}, K_{2}, K_{D}, K_{B}$ field intensity factors

$d, d_{1}$ distance from the load to crack tip

$\lambda$ relative contact zone length

l crack length

$V_{\mathrm{f}} \quad$ volume fraction of the piezoelectric inclusion

$v_{0}$ shear wave speed of the $\mathrm{BaTiO}_{3}-\mathrm{CoFe}_{2} \mathrm{O}_{4}$ composite

$k$ load ratio 
Table 1 Material properties of $\mathrm{BaTiO}_{3}$ and $\mathrm{CoFe}_{2} \mathrm{O}_{4}[40,41]$

$\left(c_{\mathrm{ij}}\right.$ in $10^{9} \mathrm{~N} / \mathrm{m}^{2}, e_{\mathrm{ij}}$ in $\mathrm{C} / \mathrm{m}^{2}, \alpha_{\mathrm{ij}}$ in $10^{-10} \mathrm{C} / \mathrm{Vm}, h_{\mathrm{ij}}$ in N/Am, $\mu_{\mathrm{ij}}$ in $10^{-6} \mathrm{Ns}^{2} / \mathrm{C}^{2}, \rho$ in $\left.\mathrm{kg} / \mathrm{m}^{3}\right)$

\begin{tabular}{ccccccccc}
\hline & $c_{11}$ & $c_{12}$ & $c_{13}$ & $c_{33}$ & $c_{44}$ & $e_{31}$ & $e_{33}$ & $e_{15}$ \\
\hline $\mathrm{BaTiO}_{3}$ & 166 & 77 & 78 & 162 & 43 & -4.4 & 18.6 & 11.6 \\
$\mathrm{CoFe}_{2} \mathrm{O}_{4}$ & 286 & 173 & 170.5 & 269.5 & 45.3 & 0 & 0 & 0 \\
\hline$h_{31}$ & $h_{33}$ & $h_{15}$ & $\alpha_{11}$ & $\alpha_{33}$ & $\mu_{11}$ & $\mu_{33}$ & $\rho$ \\
\hline $\mathrm{BaTiO}_{3}$ & 0 & 0 & 0 & 11.2 & 12.6 & 5.0 & 10.0 & 6017 \\
$\mathrm{CoFe}_{2} \mathrm{O}_{4}$ & 580.3 & 669.7 & 550.0 & 0.08 & 0.093 & 590 & 157 & 5300 \\
\hline
\end{tabular}

Table 2 Normalized critical wave speed $v_{\text {cr }} / v_{0}$ for $\mathrm{BaTiO}_{3}-\mathrm{CoFe}_{2} \mathrm{O}_{4}$ composites with different poling directions and material volume fractions

\begin{tabular}{cccccc}
\hline $\mathrm{BaTiO}_{3}-\mathrm{CoFe}_{2} \mathrm{O}_{4}$ & $\begin{array}{c}V_{\mathrm{f} 1}=0.01 \\
V_{\mathrm{f} 2}=0.99\end{array}$ & $\begin{array}{c}V_{\mathrm{f} 1}=0.1 \\
V_{\mathrm{f} 2}=0.9\end{array}$ & $\begin{array}{c}V_{\mathrm{f} 1}=0.2 \\
V_{\mathrm{f} 2}=0.8\end{array}$ & $\begin{array}{c}V_{\mathrm{f} 1}=0.3 \\
V_{\mathrm{f} 2}=0.7\end{array}$ & $\begin{array}{c}V_{\mathrm{f} 1}=0.4 \\
\beta_{0}=0, \pi\end{array}$ \\
0.9307 & 0.9345 & 0.9380 & 0.9406 & 0.9424 \\
$\beta_{0}=\pi / 12,11 \pi / 12$ & 0.8923 & 0.9004 & 0.9087 & 0.9164 & 0.9236 \\
$\beta_{0}=\pi / 6,5 \pi / 6$ & 0.8588 & 0.8703 & 0.8829 & 0.8952 & 0.9075 \\
$\beta_{0}=\pi / 4,3 \pi / 4$ & 0.8962 & 0.9053 & 0.9152 & 0.9250 & 0.9347 \\
$\beta_{0}=\pi / 3,2 \pi / 3$ & 0.9317 & 0.9372 & 0.9428 & 0.9475 & 0.9474 \\
$\beta_{0}=5 \pi / 12,7 \pi / 12$ & 0.8857 & 0.8933 & 0.9014 & 0.9091 & 0.9165 \\
$\beta_{0}=\pi / 2$ & 0.8512 & 0.8606 & 0.8707 & 0.8806 & 0.8904 \\
\hline
\end{tabular}


(a)

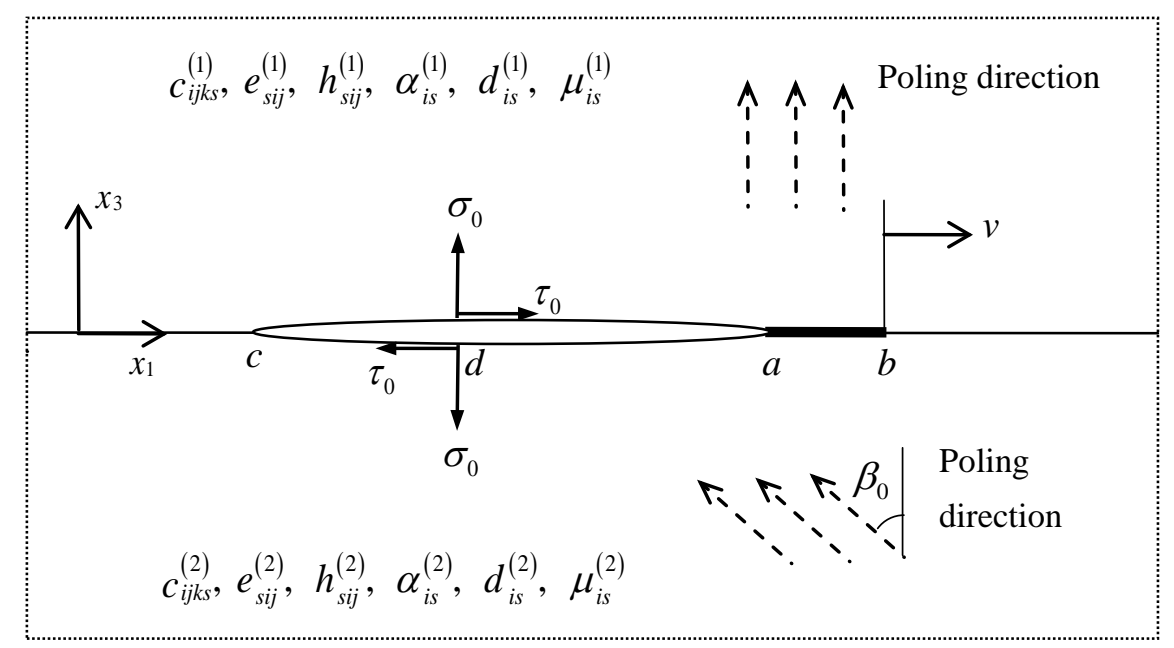

(b)

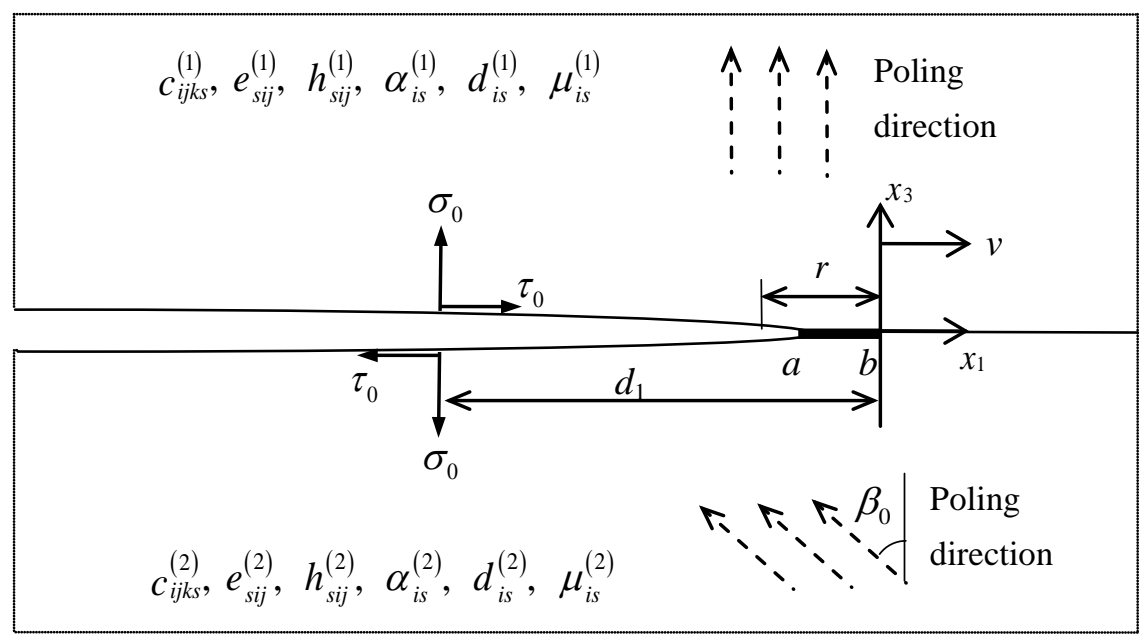

Fig. 1. Moving crack with frictionless contact zone at interface of MEE bimaterial under concentrated loading: (a) finite crack, and (b) semi-infinite crack 


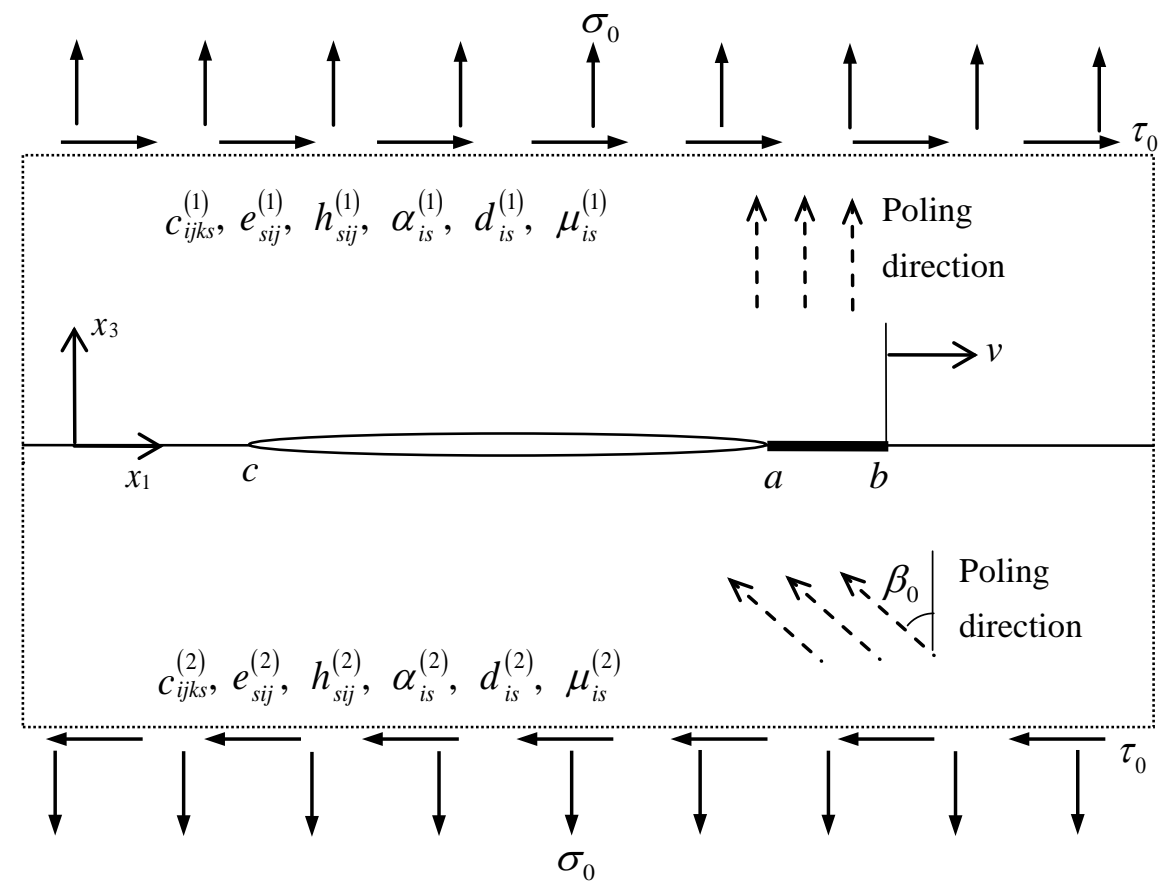

Fig. 2. Moving crack with frictionless contact zone at interface of MEE bimaterial under uniform mixed-mode loading 
(a)

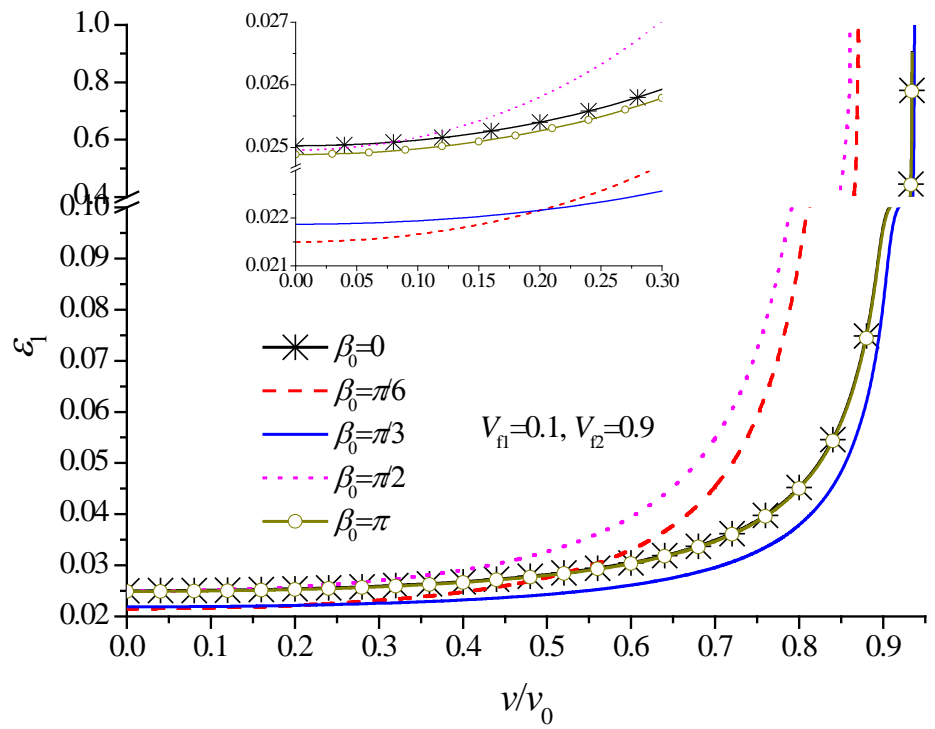

(b)

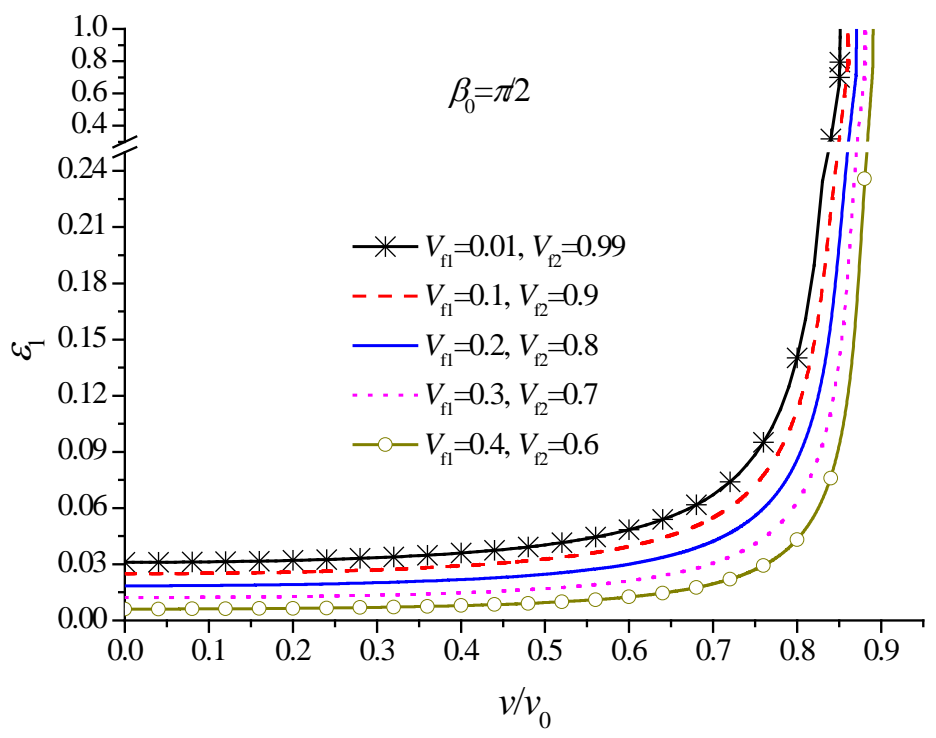

Fig. 3. Variation of the oscillating index $\varepsilon_{1}$ with respect to normalized speed of the moving crack $v / v_{0}$ for different (a) poling directions, and (b) material volume fractions 
(a)

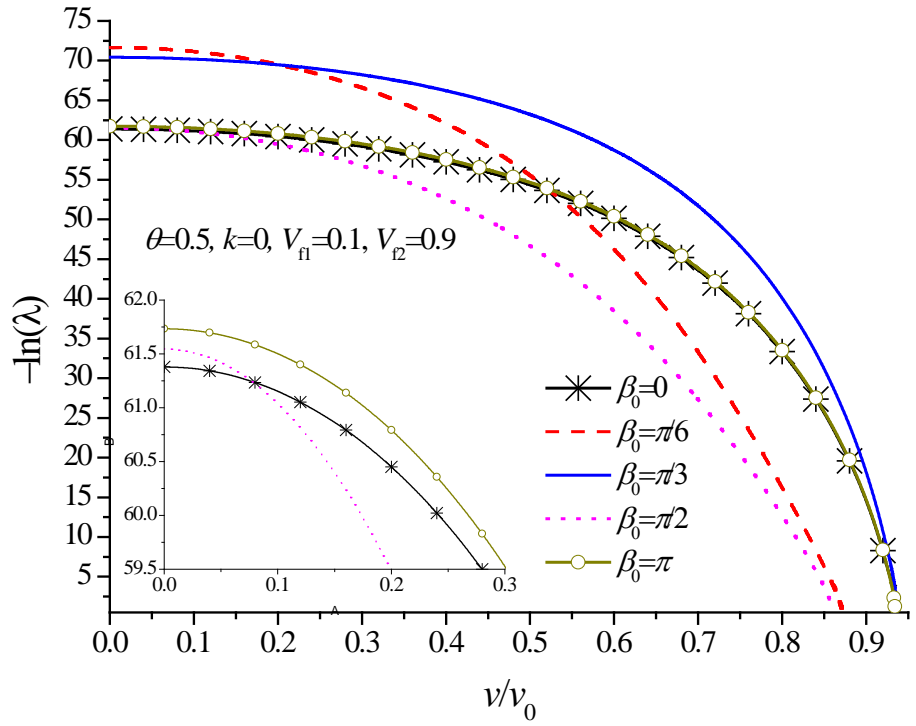

(b)

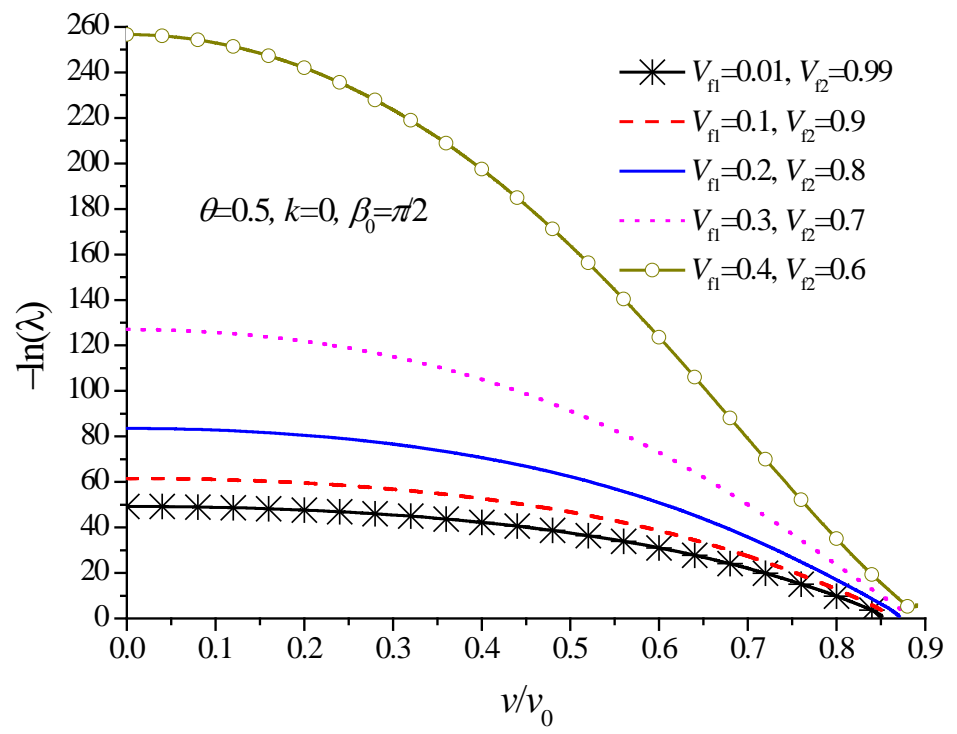

Fig. 4. Relative length of contact zone $\lambda$ of finite crack with respect to normalized speed of moving crack $v / v_{0}$ for different (a) poling directions, and (b) material volume fractions under concentrated loading with $k=0$ 

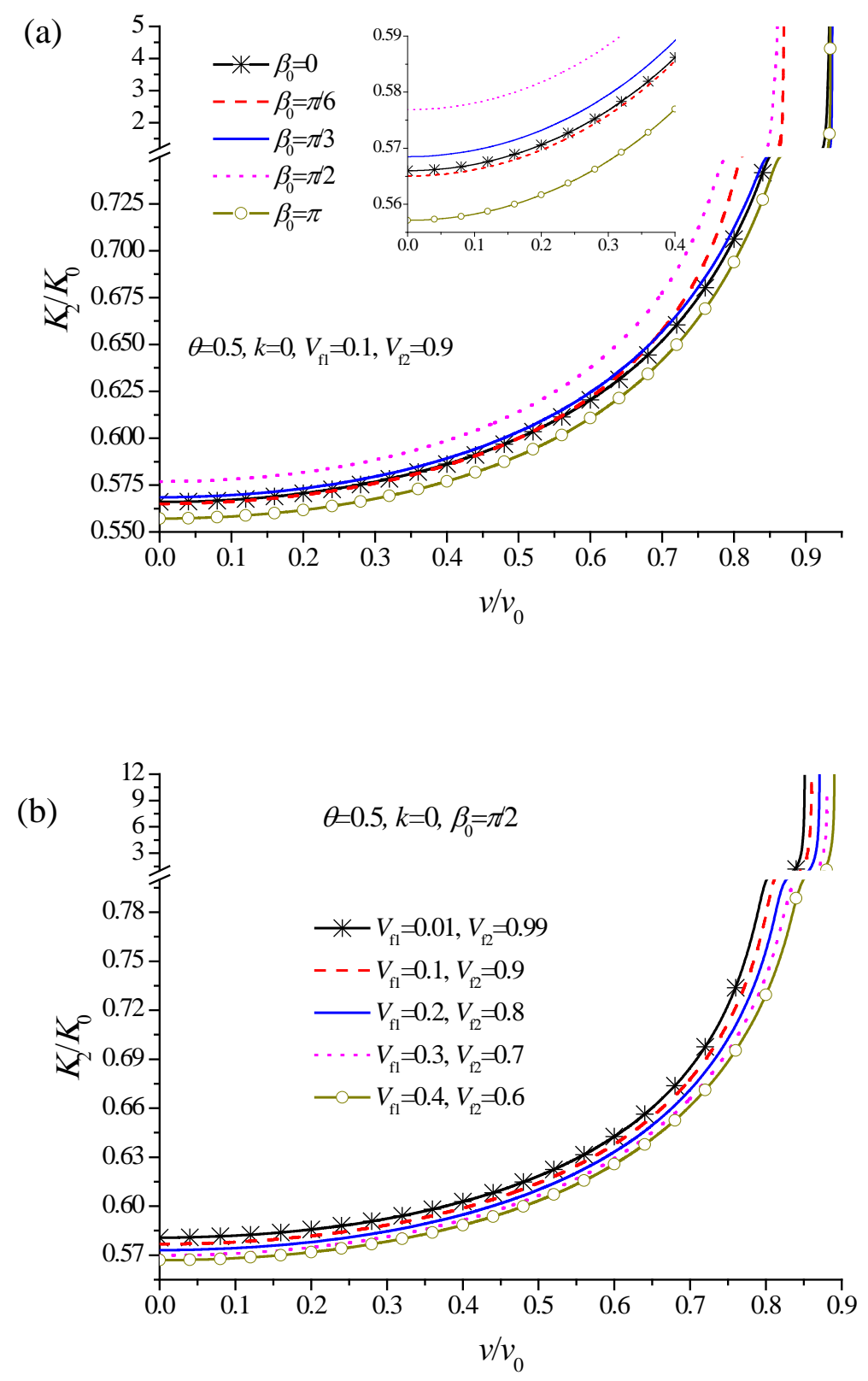

Fig. 5. Normalized FIF $K_{2} / K_{0}$ of finite crack with respect to normalized speed of moving crack $v / v_{0}$ for different (a) poling directions, and (b) material volume fractions under concentrated loading with $k=0$ 
(a)

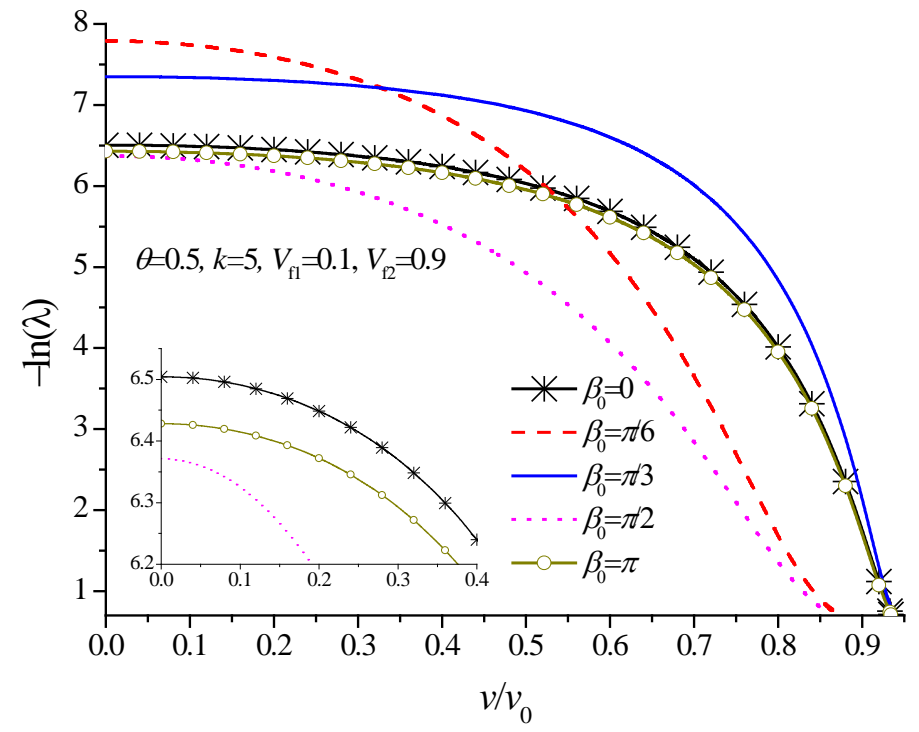

(b)

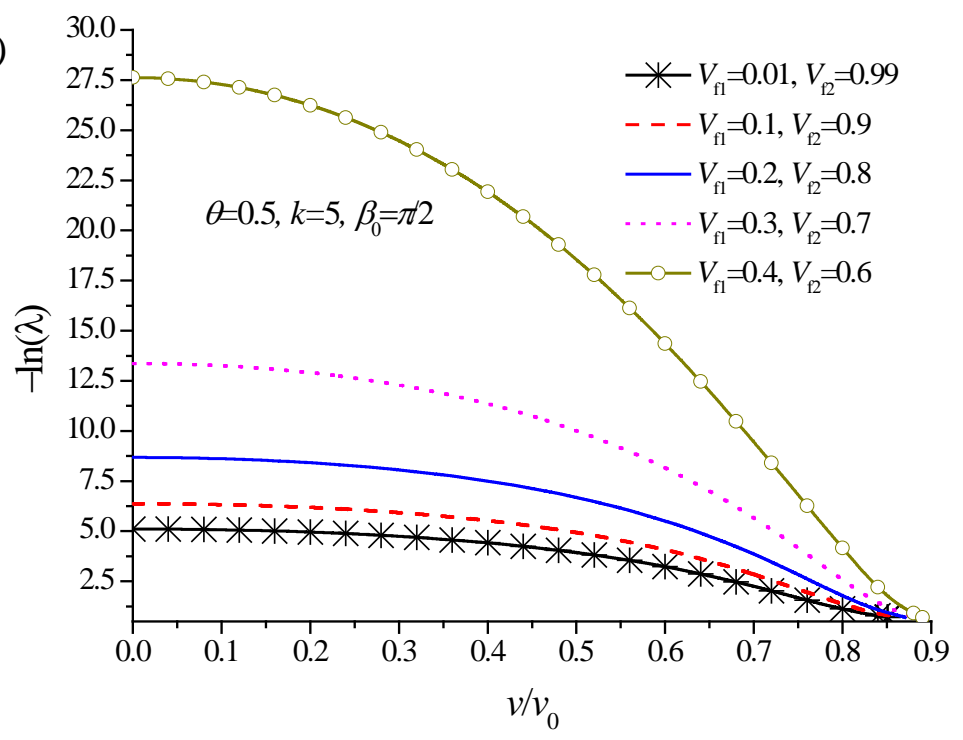

Fig. 6. Relative length of contact zone $\lambda$ of finite crack with respect to normalized speed of moving crack $v / v_{0}$ for different (a) poling directions, and (b) material volume fractions under concentrated loading with $k=5$ 

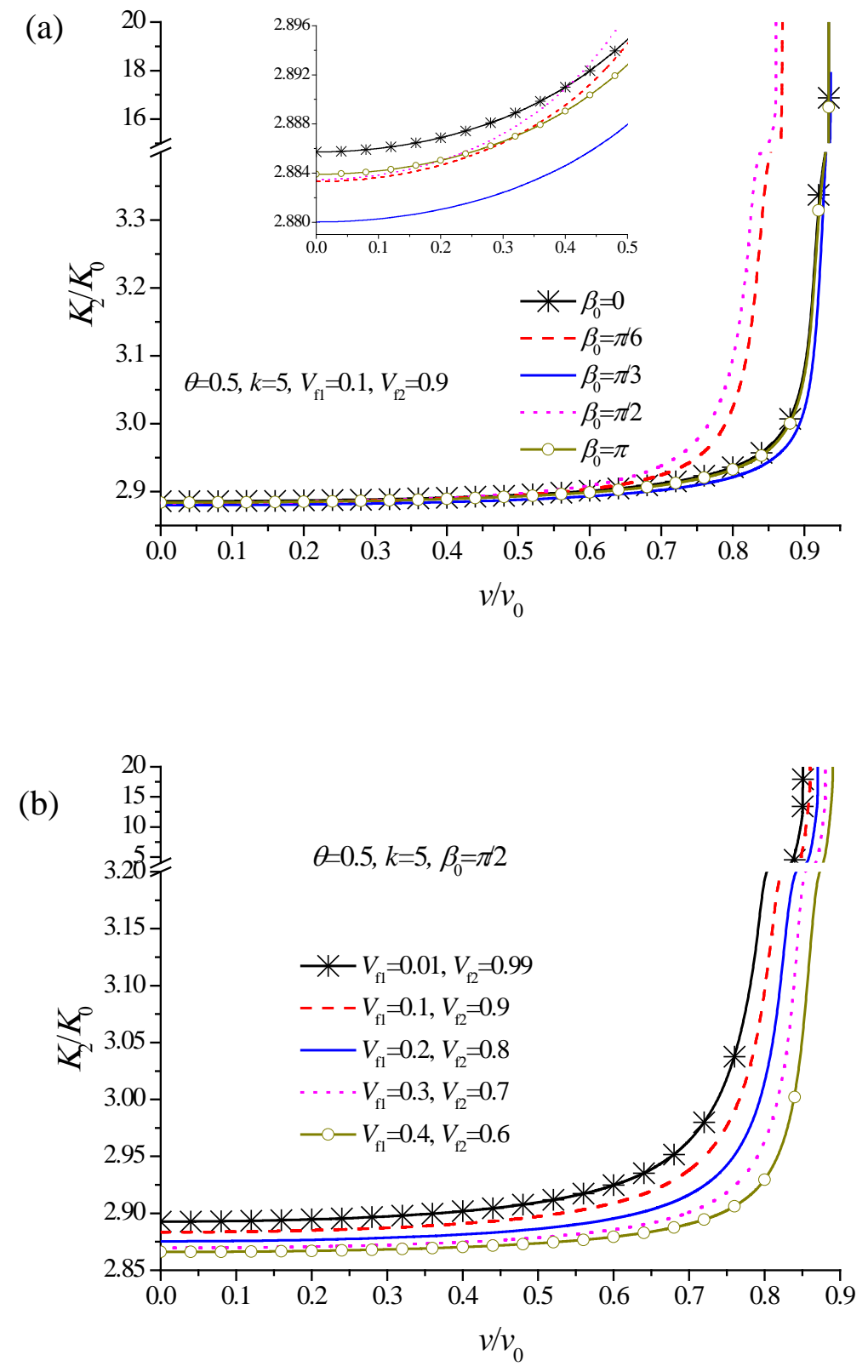

Fig. 7. Normalized FIF $K_{2} / K_{0}$ of finite crack with respect to normalized speed of moving crack $v / v_{0}$ for different (a) poling directions, and (b) material volume fractions under concentrated loading with $k=5$ 


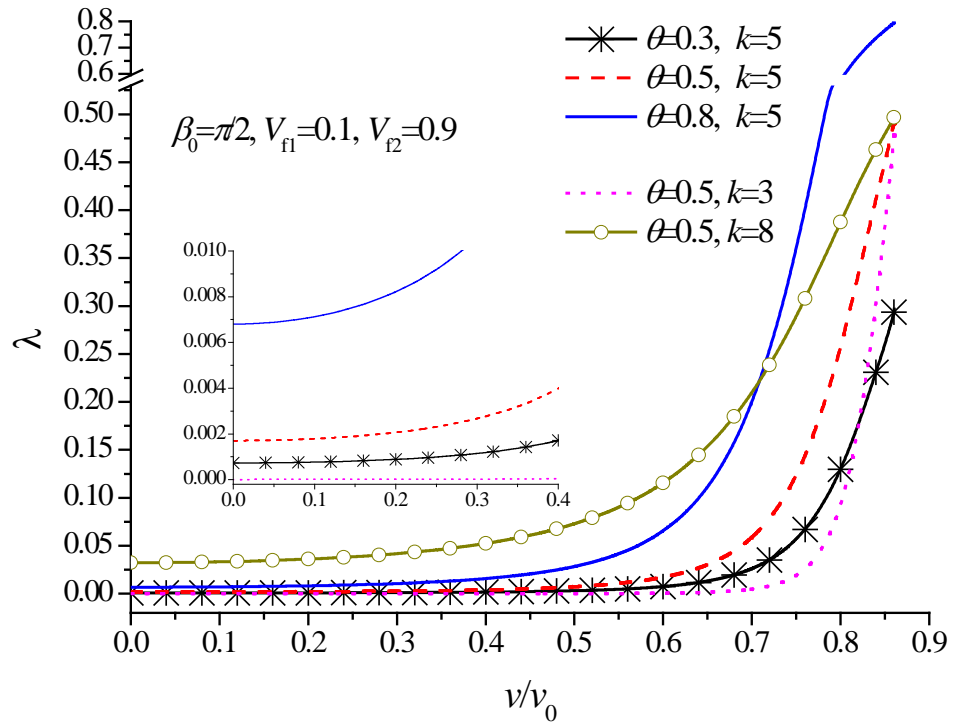

Fig. 8. Relative length of contact zone $\lambda$ of finite crack with respect to normalized speed of moving crack $v / v_{0}$ for different load positions and load ratios under concentrated loading 


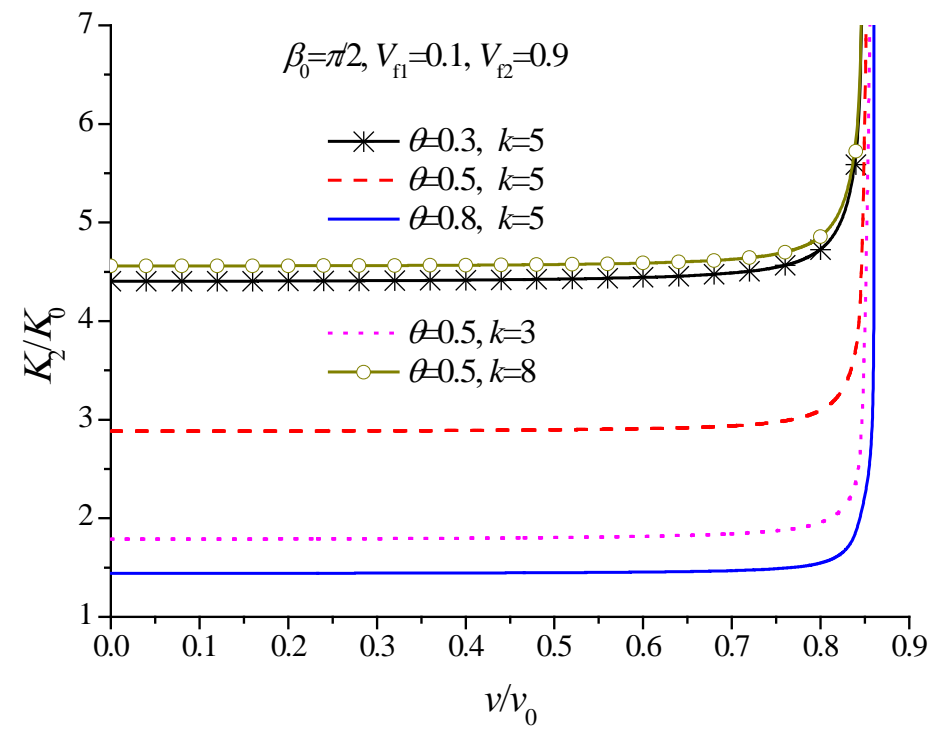

Fig. 9. Normalized FIF $K_{2} / K_{0}$ with respect to normalized speed of moving crack $v / v_{0}$ for different load positions and load ratios under concentrated loading 


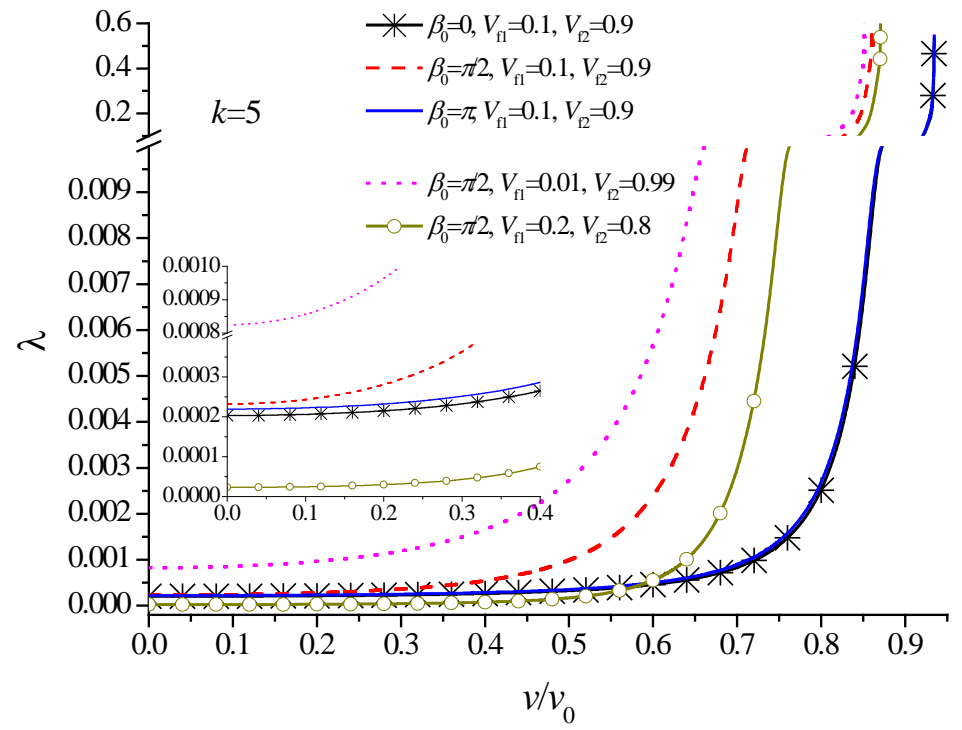

Fig. 10. Relative length of contact zone $\lambda$ of finite crack with respect to normalized speed of moving crack $v / v_{0}$ for different load positions and load ratios under uniform mixed-mode loading 


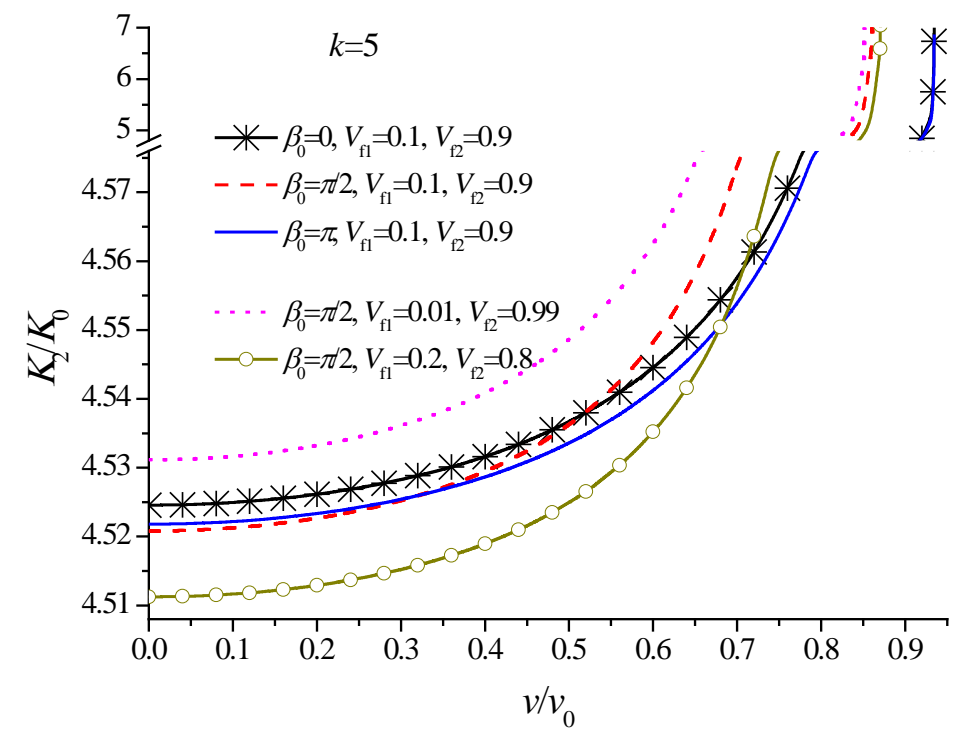

Fig. 11. Normalized FIF $K_{2} / K_{0}$ of finite crack with respect to normalized speed of moving crack $v / v_{0}$ for different load positions and load ratios under uniform mixed-mode loading 